\title{
Genome-wide discovery, and computational and transcriptional characterization of an AIG gene family in the freshwater snail Biomphalaria glabrata, a vector for Schistosoma mansoni
}

\author{
Lijun Lu', Eric S. Loker', Si-Ming Zhang ${ }^{1}$, Sarah K. Buddenborg² and Lijing Bu ${ }^{1 *}$ (D)
}

\begin{abstract}
Background: The AIG (avrRpt2-induced gene) family of GTPases, characterized by the presence of a distinctive AIG1 domain, is mysterious in having a peculiar phylogenetic distribution, a predilection for undergoing expansion and loss, and an uncertain functional role, especially in invertebrates. AIGs are frequently represented as GIMAPs (GTPase of the immunity associated protein family), characterized by presence of the AIG1 domain along with coiled-coil domains. Here we provide an overview of the remarkably expanded AIG repertoire of the freshwater gastropod Biomphalaria glabrata, compare it with AIGs in other organisms, and detail patterns of expression in B. glabrata susceptible or resistant to infection with Schistosoma mansoni, responsible for the neglected tropical disease of intestinal schistosomiasis.
\end{abstract}

Results: We define the 7 conserved motifs that comprise the AIG1 domain in B. glabrata and detail its association with at least 7 other domains, indicative of functional versatility of B. glabrata AIGs. AIG genes were usually found in tandem arrays in the B. glabrata genome, suggestive of an origin by segmental gene duplication. We found 91 genes with complete AIG1 domains, including 64 GIMAPs and 27 AIG genes without coiled-coils, more than known for any other organism except Danio (with > 100). We defined expression patterns of AlG genes in 12 different $B$. glabrata organs and characterized whole-body AIG responses to microbial PAMPs, and of schistosome-resistant or -susceptible strains of B. glabrata to S. mansoni exposure. Biomphalaria glabrata AIG genes clustered with expansions of AIG genes from other heterobranch gastropods yet showed unique lineage-specific subclusters. Other gastropods and bivalves had separate but also diverse expansions of AIG genes, whereas cephalopods seem to lack AIG genes.

Conclusions: The AIG genes of B. glabrata exhibit expansion in both numbers and potential functions, differ markedly in expression between strains varying in susceptibility to schistosomes, and are responsive to immune challenge. These features provide strong impetus to further explore the functional role of AIG genes in the defense responses of B. glabrata, including to suppress or support the development of medically relevant S. mansoni parasites.

Keywords: AIG gene, AIG1 domain, GIMAP, IAN, Coiled-coil, Conserved motif, Biomphalaria glabrata, Schistosoma mansoni, Mollusca, Invertebrate, Gene expression

\footnotetext{
* Correspondence: lijing@unm.edu

${ }^{1}$ Center for Evolutionary and Theoretical Immunology, Department of

Biology, University of New Mexico, Albuquerque, NM 87131, USA

Full list of author information is available at the end of the article
}

(c) The Author(s). 2020 Open Access This article is distributed under the terms of the Creative Commons Attribution 4.0 International License (http://creativecommons.org/licenses/by/4.0/), which permits unrestricted use, distribution, and reproduction in any medium, provided you give appropriate credit to the original author(s) and the source, provide a link to the Creative Commons license, and indicate if changes were made. The Creative Commons Public Domain Dedication waiver (http://creativecommons.org/publicdomain/zero/1.0/) applies to the data made available in this article, unless otherwise stated. 


\section{Background}

Characterization of the immune defense capabilities of invertebrates has been aided by the increasing number of available genomes, more comprehensive transcriptional studies that outline invertebrate responses to a variety of pathogens, and by the rapidly growing availability of bioinformatics tools to enable analysis and comparison of such responses [1-3]. Invertebrate defenses are complex and often involve deployment of unexpectedly large families of immune-related molecules [4-6]. In addition to large gene families the individual members of which might be expressed in specific ways following particular kinds of stimuli $[5,6]$, other mechanisms to diversify invertebrate responses such as allelic diversity, alternative splicing and somatic recombination have been reported, adding to the potential of invertebrates to fine-tune their responses to pathogens [7-10]. Additionally, different invertebrate groups may be challenged by distinctive kinds of infectious agents, as for example from particular groups of fungal or metazoan parasites with which they are regularly exploited and with which they have co-evolved [11]. Consequently, the overall array of invertebrate responses becomes very impressive.

The study of invertebrate immunity has been aided by investigations of the defense responses of plants and vertebrates, and vice versa [12]. One example of a group of immune-related molecules first discovered in plants and mammals is the AIG family of GTPases. The first family member, AIG1 (avrRpt2-induced gene), was discovered in Arabidopsis thaliana and its expression was induced by exposure to plant pathogens or abiotic stressors [13-15]. The AIG1 domain consists of G1 through G5 boxes and two unique conserved motifs, the consensus box CB located between G3 and G4, and the IAN (immune-associated nucleotide-binding protein) consensus sequence that partially overlaps the G5 region. The AIG1 domain comprises a GTP binding region, and genes containing an AIG1 domain are called AIG genes. In plants and vertebrates, the AIG family of GTPases is frequently represented by GIMAPs (GTPase of the immunity associated protein family), also known as immune-associated nucleotide binding proteins or IANs. GIMAPs are proteins of $30-80 \mathrm{kDa}$ containing coiled-coil regions along with a characteristic AIG1 domain. Some GIMAP genes encode proteins with membrane-anchoring domains whereas others are soluble proteins [16].

In mammals where they have been most extensively studied, GIMAPs are involved in regulating and maintaining $\mathrm{T}$ cell numbers and survival $[17,18]$. They are associated with both proliferative and apoptotic processes [16]. GIMAPs are also known from the coral Acropora millepora for which a role for GIMAPs in phagolysosomal processing was proposed [19]. In support of this idea, GIMAPs found in Lewis rats resistant to the apicomplexan parasite Toxoplasma gondii have been implicated in binding to the parasitophorous vacuole surrounding these intracellular parasites and favoring fusion with host cell lysosomes, thereby leading to the demise of the parasites [20]. GIMAPs may be linked to clinically relevant phenomena like T-cell leukopenia autoimmunity or leukemia.

One of the most interesting aspects of GIMAP biology is their peculiar phylogenetic distribution. They are found in plants [21], some protists like Entamoeba [22], corals but not all cnidarians [19], gastropod [23-25] and bivalve molluscs $[5,23,26]$, in the cephalochordate Branchiostoma (lancets) and the hemichordate Sacoglossus (acorn worm) and in vertebrates [19, 23]. They are lacking as far as is known in representative fungi like yeast like Saccharomyces cerevisae, early diverging metazoans such as the placozoan Trichoplax or the sponge Amphimedon, or in the model ecdysozoans Caenorhabditis elegans or Drosophila melanogaster. A recent study predicted 60 AIG1 genes in the genome of the subterrestrial, thermally-stressed nematode Halicephalobus mephisto [27]. In deuterostomes, they are not known from the tunicate Ciona, the sea urchin Strongylocentrotus or from lampreys [19, 23].

With respect to numbers of GIMAP loci, Arabidopsis has 13 [28], the oyster Crassostrea virginica has 28 [23], and zebrafish have over 100 [19]. Rats have 7 genes, humans 8 and mice $9[29,30]$. AIG gene family expansions have occurred in bivalves and in the nematode $H$. mephisto [19, 23, 27]. GIMAP loci are clustered in plants, corals and mammals, suggestive of tandem gene duplications [16, 23, 28, 29]. The phylogenetic distribution is consistent with an ancient origin for GIMAPs accompanied by independent losses in some lineages and amplifications in others $[19,23,31]$. A note of caution has been expressed that the similarities between plant IANs and animal GIMAPs may represent convergence [19].

Our interest in AIG genes is centered on planorbid gastropods in the genus Biomphalaria, particularly the Neotropical species B. glabrata and African species such as $B$. pfeifferi. These snails serve as vectors of the human parasite Schistosoma mansoni. Schistosomes are responsible for schistosomiasis, a neglected tropical disease that still infects over 200 million people [32, 33]. In a microarray-based study of the transcriptomic responses of the hematopoietic organ of B. glabrata, four GIMAPs were found to be significantly up-regulated following exposure to bacterial lipopolysaccharide (LPS) and peptidoglycan (PGN) [24]. An RNA-Seq study of the transcriptomic responses of B. pfeifferi to $S$. mansoni revealed that GIMAPs were up-regulated at both one and three days post-exposure [25]. Given the presence of GIMAPs in B. glabrata, their responsiveness to immune stimuli including schistosomes, and the association of GIMAPs with immune cell numbers and regulation noted in other model systems, we undertook 
a further examination of the AIG gene family in B. glabrata. Our studies are motivated by the need to develop novel methods of schistosome control based on development of snails resistant to schistosome infection.

\section{Results}

The following definitions are used throughout the paper: AIG1 domain: a conserved domain including G1-G4, G5/IAN motifs and a conserved box (CB) found with the predicted protein sequences; partial AIG gene: gene containing an incomplete AIG1 domain, with absence of at least one conserved motif; AIG gene: gene containing at least one complete AIG1 domain, and possibly other domains; B. glabrata GIMAP: B. glabrata gene containing a complete AIG1 domain and one or more coiledcoil domains. We refer to the more formal names for designated genes like "BGLB008770" as "Bg8770" for the sake of readability. The "-PB" or "-RB" suffix following the gene ID is the protein ID or transcript ID of the corresponding gene, respectively.

\section{Conserved motifs within the $B$. glabrata AIG1 domain} Conserved motifs (G1-G4, G5/IAN and CB) within the AIG1 domains of B. glabrata are indicated in Fig. 1, with more details in Additional file 2: Table S1, and occur in the order expected. In addition to consensus signatures, the motifs also contain some unique sequences as compared to known motif variants in AIG1 domains from other organisms. Motifs G1-G3 in B. glabrata are similar to those originally defined from human sequences. For example, protein sequences like GxxxxGKS, the conserved G1 motif in other organisms, can also be found near the beginning of the B. glabrata AIG1 domain sequence. Multiple sites of similar conservation within the G1 motif such as GKTGxGKS can also be found. Conserved sequences flanking the G1 motif in B. glabrata are also observed, such as LLLT/V on the N-terminal and A/STGNS/T on the C-terminal sides. Similarly, G2 and G3 motifs in $B$. glabrata are SxTx and DxPG (Fig. 1). The CB, G4 and G5/IAN motifs in B. glabrata exhibit some variation compared to other organisms, but still can be accurately located by reference to known consensus sequences for the motifs, their relative location and their flanking conserved sequences. The $\mathrm{CB}$ consensus sequence in B. glabrata $\mathrm{xC/}$ NPxxxGxxAxLLVLKYGxRFxxTxEE (Fig. 1) has the most similarity to the mouse counterpart (LSxPGPHALLLV xQLG-RF/Y TxE D/E), which can be found near the Cterminus of the G3 motif. The G4 motif in B. glabrata (TxGD) has consensus sequence variations of NKxD in human, mouse and plant, and $\mathrm{TxCD} / \mathrm{E}$ in coral. The G5/ IAN motif in B. glabrata has a RxVLF D/N N signature, which is partially overlapping with the mouse IAN motif RxxxFNN K/R AxxxE. In the mouse AIG1 domain, the G5 motif is embedded in IAN ( $x x x$ in RxxxFNN), while the corresponding location in B. glabrata contains a less conservative sequence (amino acids of top frequencies are C28\%, V60\%, L86\%), resulting in "xVL" in RxVLF D/N N).

\section{Overview of the B. glabrata AIG gene family}

An initial scan with the HMM AIG1 domain profile (PFAM ID: PF04548) returned genes containing AIG1 domains as well as domains belonging to other GTPase families within the same P-loop NTPase superfamily. All non-AIG1 GTPase genes were filtered out after scanning

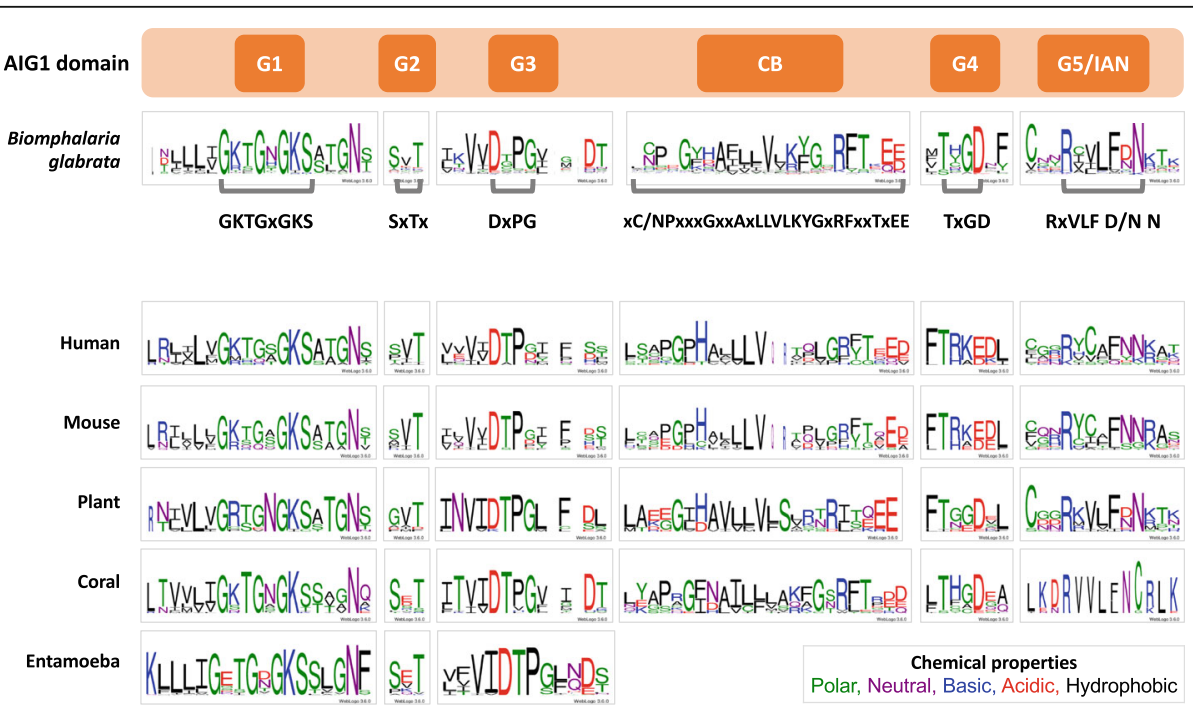

Fig. 1 Conserved motifs within the B. glabrata AIG1 domain. Accurate locations of conserved AlG1 motifs (G1 = GKTGXGKS; G2 = SxTx; G3 = DxPG; $\mathrm{CB}=x \mathrm{C} / \mathrm{NP} x x x \mathrm{G} x x$ AxLLLVLKYGxRFxxTxEE; G4 = TxGD; G5/IAN = RxVLF D/N N) in B. glabrata were marked out with grey bars under each logo. Consensus sequences for AIG1 domains in other organisms were collected from published literature: human (Homo sapiens) [29], mouse (Mus musculus) [16], plant (Arabidopsis thaliana) [28], coral (Acropora millepora) [19], and entamoeba (Entamoeba histolytica) [22] 
with the InterProScan profile. In total we found 111 genes (148 predicted proteins) with complete or partial AIG1 domains (Additional file 3: Table S2). Of these, 91 genes (128 proteins) had complete AIG1 domains, and the remainder had AIG1 domains missing G1, G5/IAN or additional motifs and were considered to be partial AIGs.

The 91 complete AIG genes exhibit 19 different arrangements of domain architectures based on predictions using InterProScan (Fig. 2). Taking into account alternative splicing, an additional five domain architectures were found. For example, gene $B g 8770$ has three splice variants: DDCARD + AIG1 + coiled-coil (Bg8770-RB), AIG1 + coiled-coil $($ Bg8770-RC) and DD-CARD + ARM-type-fold + AIG1 + coiled-coil (Bg8770-RD).

There are 64 genes (101 proteins) with an AIG1 domain and at least one predicted coiled-coil, meaning they fit the criteria associated with a B. glabrata GIMAP. For the following analyses we also considered the 27 AIG-containing genes without coiled-coils because: a) the evolutionary history of GIMAP genes is likely to be entwined with AIG genes lacking coiled-coils; and b) coiled-coil domains can be missed by prediction tools because they vary considerably in length $(87 \sim 3000$ aa) and degree of sequence conservation (from 29.4\% to hypervariable 97.1\%) [34]. Coiled-coil domains could be identified on either side of AIG1 domains, indicating the possibility of polymerization, possibly influencing ligand binding [16, 35]. Detailed predictions for classification as antiparallel and parallel dimers, trimers and tetramers based on LOGICOIL are listed in Additional file 3: Table S2.

We also found genes containing unusual or complex domain architectures including dual AIG1 domains, an AIG1 domain with additional $\mathrm{N}$-terminal death domains (DD) or protein kinase (Pkinase) domains, with an $\mathrm{N}$ terminal Armadillo (ARM)-type fold, or a C-terminal hint/ hedgehog domain (Fig. 2). Because of variable domain architectures, AIG proteins were predicted to range from 105 aa $(19-\mathrm{kDa})$ for incomplete AIG1 domains to 1286 aa (141$\mathrm{kDa})$.

\section{Transmembrane predictions for the AIG family}

No signal peptide was encoded by any of the AIG genes. Additionally, 12 AIG genes contained predicted transmembrane domains (TM), suggestive of their location on the plasma membrane or intracellular membranes. A further look based on TMHMM results revealed interesting differences in membrane spanning structures (Fig. 3). TM domain numbers ranged from 1 to 3 , with at least three types of structures noted. Additionally, the AIG1 domain can be on either side of the membrane, adding an extra layer of potential functionality. Lastly, 10 out of 12 TM domain-containing AIG genes are associated with coiled-coils, indicating the possibility of multimerization on the membrane.

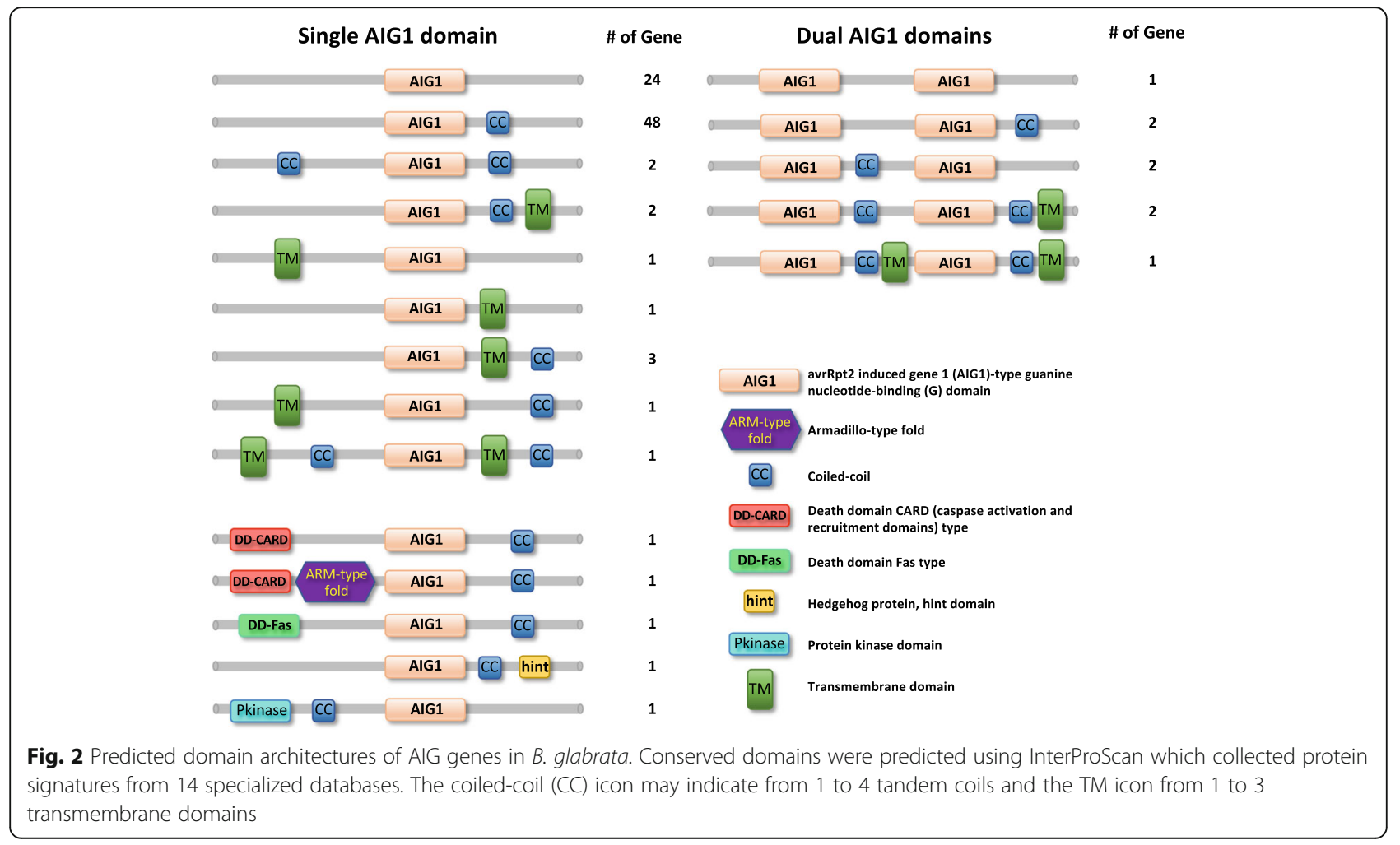



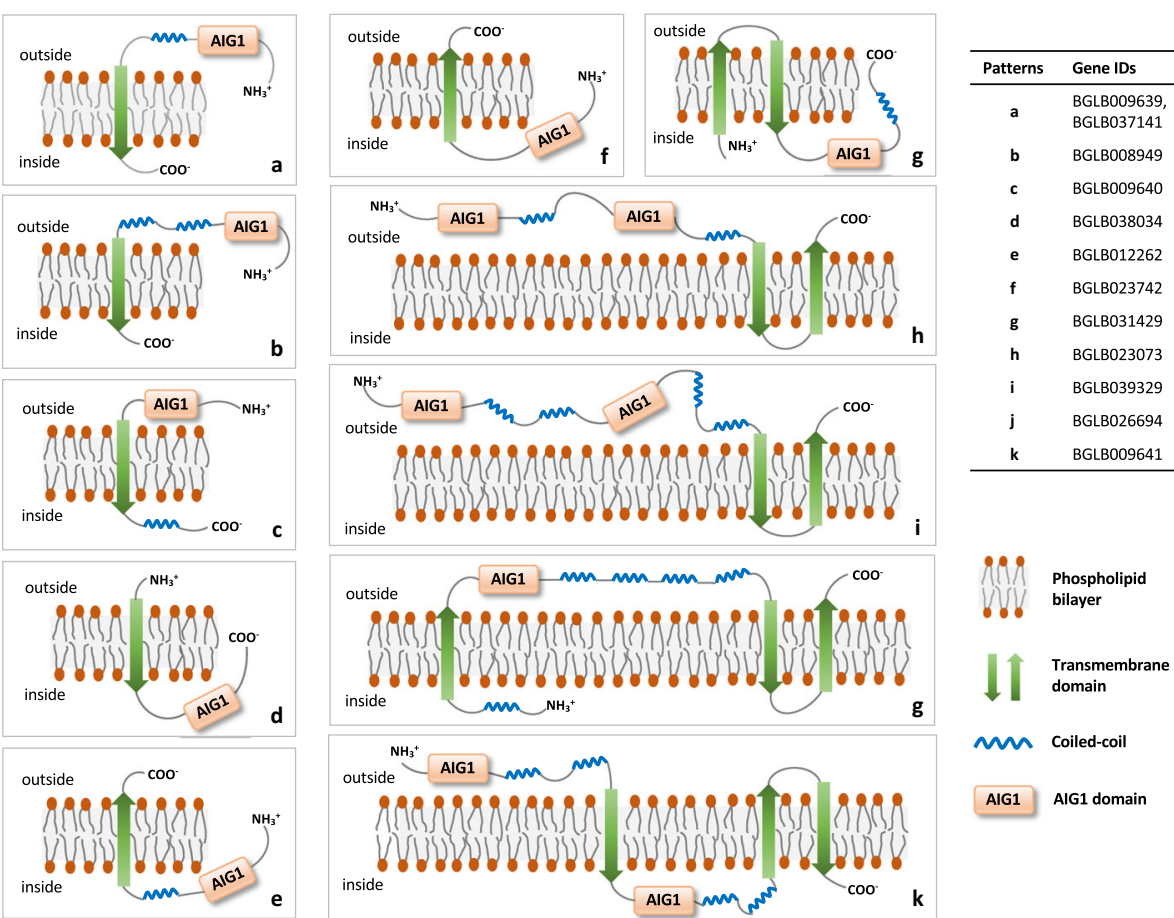

Fig. 3 Hypothetical transmembrane (TM) dispositions of predicted polypeptides of the AIG genes of B. glabrata. The phospholipid bilayers here could represent not only plasma membranes, but also could be membranes of organelles including mitochondria, endoplasmic reticulum (ER), Golgi apparatus or other membranous organelles. There are 3 types of hypothetical TM dispositions: Type I, a single transmembrane span with Nterminus on the outside (a-d); Type II, a single transmembrane span, with C-terminus on the outside (e-f); and Type III, with multiple spans (g-k). Some models predict the presence of an AIG1 domain on both side of a membrane

\section{Scaffold locations and arrangements of AIG genes in $B$. glabrata}

We included both complete and partial AIG genes in this analysis. The reference genome of $B$. glabrata BB02 strain contains 331,400 scaffolds, 13,826 of which have been annotated. The AIG footprints are located on 66 different scaffolds (Additional file 1: Fig. S1), thirteen of which contain at least two complete or partial AIG genes. Tandem arrays of complete or partial AIGs were found on 12 scaffolds (Fig. 4). For example, on Scaffold 39, there are 11 AIG genes forming one tandem array, 10 of which are B. glabrata GIMAPs. Similarly, on Scaffold 334, there are two tandem arrays with 9 AIG genes, 8 of which are GIMAPs. A total of 50 AIG footprints (31 GIMAPs, 9 AIG genes without coiled-coils, and 10 partial AIGs) were found to in tandem arrays ranging 2 to 11 genes. There are three orientation types among the tandem gene pairs: 1) parallel $\rightarrow \rightarrow$ or $\leftarrow \leftarrow$ (16 pairs); 2$)$ convergent $\rightarrow \leftarrow(10$ pairs); and 3) divergent $\leftarrow \rightarrow$ (12 pairs). In Fig. 4 , the gray brackets on some scaffolds show the AIG genes in tandem array. Additionally, 55 genes are dispersed on the other 54 scaffolds. For example, Scaffold 43 contains two GIAMPs separated by $400 \mathrm{~kb}$ (Additional file 3: Table S2, Additional file 1: Fig. S1).

\section{AIG gene expression analysis in Biomphalaria spp} I). Constitutive expression in different snail organs [36] Organ specific gene expression was assessed using RNASeq data from 12 organs of unstimulated B. glabrata BB02 strain snails [36]: buccal mass, kidney, heart, central nervous system, digestive gland, ovotestes, stomach, albumen gland, terminal genitalia, head foot, mantle edge, and salivary gland. Based on transcripts per million (TPM) transformed Z scores, 47 GIMAPs and 11 additional AIG genes showed significant gene expression (Fig. 5, and Additional file 5: Table S4 for domain and other features). The most highly expressed transcripts were found in stomach, digestive gland and terminal genitalia. Each organ had a specific pattern of AIG gene expression, but some pairs of organs were more similar to each other than to others (e.g. stomach and kidney, or albumen gland and terminal genitalia). There were also several "clusters" of transcripts with similar expression patterns among the organs. In digestive gland, 10 transcripts (encoded by 1 AIG and 8 GIMAP genes) were preferentially expressed and clustered together, four of them with transmembrane domains. In stomach, 9 GIMAP transcripts originating from widely distributed scaffolds were overexpressed, of which two have dual AIG1 domains and one a transmembrane domain. In the salivary glands, 8 


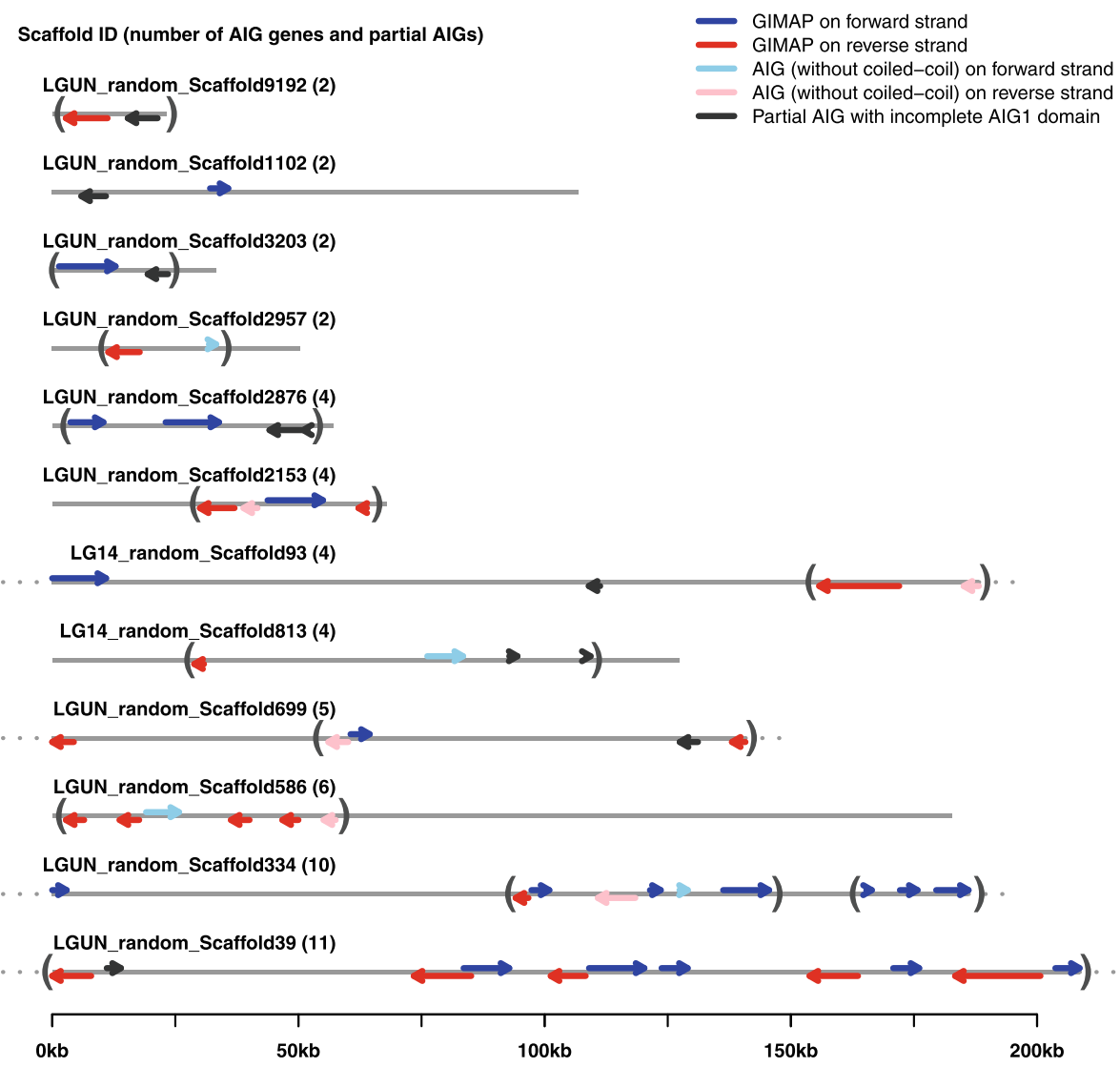

Fig. 4 Scaffold locations of evolutionary footprints of AIG genes in the B. glabrata BB02 genome. The evolutionary footprints of AIG genes in B. glabrata consist of three types: GIMAP (AIG gene with coiled-coil domain), AIG gene without coiled-coil domain, and partial AIGs. Scaffold backbones were drawn with gray lines. Scaffolds longer than the figure region were marked with gray dots on left or right end of the gray lines. Genes on the forward strand were marked out using left-to-right arrows above scaffold lines, showing GIMAP genes (blue) and AIG genes (sky blue). Genes on the reverse strand were marked out using right-to-left arrows below scaffold lines, showing GIMAP genes (red) and AIG genes (pink). Partial AIGs (black) were showing on both forward and reverse strands. Scaffold IDs were labeled above each scaffold. Numbers in parenthesis after scaffold IDs are total number of AIG genes (with and without coiled-coils) on the scaffold. Gray parentheses enclosed genes within the same tandem array (no other genes in between)

transcripts (encoded by 7 GIMAPs) were under-expressed, one of which has hint/hedgehog domains.

\section{II). Analysis of AIG gene expression from previously published microarray study of B. glabrata: responses to immunogens LPS (lipopolysaccharide), PGN (peptidoglycan) or FCN (fucoidan) [24]}

Gene expression values (Fig. 5, Additional file 5: Table S4) of the schistosome resistant BS-90 strain of B. glabrata indicated that four GIMAP genes (Bg9640, Bg11834, Bg25758 and Bg21576) were significantly upregulated (from 3 - to 13 -fold) following injection with LPS [24]. The first two are categorized as B. glabrata GIMAPs, with Bg9640 having a transmembrane domain (see also Fig. 3b). Bg9640 was also highly expressed in digestive gland and mantle edge (Fig. 5). The third gene Bg25758 identified by Zhang et al. [24] lacked G1-G3 motifs and coiled-coils. Their fourth gene originally annotated as a GIMAP we reannotated as "non-coding RNA" based on NCBI gene bank (XR_001217856.1) and VectorBase v1.6 entries.

We also discovered 10 more AIG genes match probes on the DE genes list in the microarray study (Fig. 5, Additional file 5: Table S4). All 10 genes were initially annotated as "NA" (no Genbank match) but based on our AIG criteria they include 7 GIMAPs, 2 complete AIG, and 1 partial AIG (Additional file 5: Table S4). One of the GIMAP genes (Bg17413) was significantly up-regulated in snails exposed to LPS (18.6 fold) or to PGN (5.7 fold). Conversely, the GIMAP gene Bg10064 was down-regulated 2.4 fold following FCN treatment. FCN is a complex polysaccharide derived from the brown alga Fucus vesiculosus and is thought to mimic fucosyl-rich glycans found on the surfaces of sporocysts of S. mansoni [24]. 


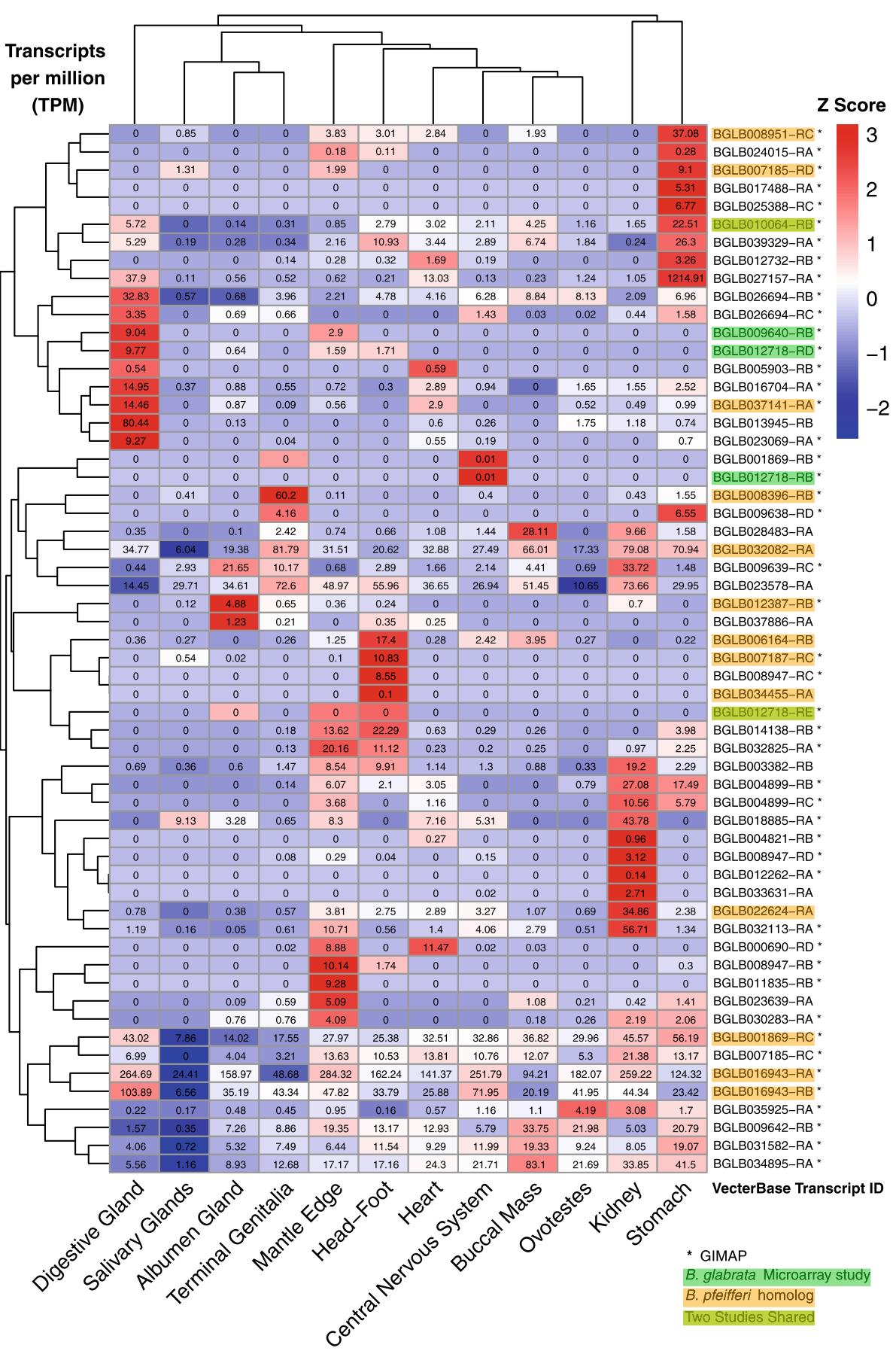

Fig. 5 Heatmap of organ specific expression of B. glabrata AIG genes. RNA-Seq reads from 12 organs of B. glabrata BB02 [36] were analyzed. Blocks in the heat map were colored in reference to Z scores transformed from transcripts per million (TPM). Z scores were calculated as (TPM mean across organ)/standard deviation across organs. The $Z$ score is a cross-organ normalization of TPM for each individual gene, and for a given gene, Z scores among organs are comparable. To compare gene expression within one specific organ, TPM was used. VectorBase transcript IDs were used to match with a specific AIG gene on each row. Those IDs with an asterisk are B. glabrata GIMAPs. IDs in green were target sequences of microarray probes in the study on gene expression of B. glabrata BS-90 strain (schistosome-resistant) injected with pathogens [24]. IDs in orange were homologs of AIG genes (cut-off values: > 70\% identity; > 90\% coverages) for a related snail, B. pfeifferi, from RNA-Seq data [25]. IDs in mustard color were AIG genes that appeared in both studies above 
III). AIG analysis from previously published RNA-Seq results obtained for field-derived specimens of the African schistosome vector snail Biomphalaria pfeifferi exposed to S. mansoni [25]

Biomphalaria pfeifferi is a widely distributed and important vector for S. mansoni in sub-Saharan Africa, and is a close relative of B. glabrata. A total of $28 \mathrm{~B}$. pfeifferi homologs for $B$. glabrata AIG genes were identified using a BLASTp search cutoff of $70 \%$ identity and $90 \%$ alignment coverages on both query (translated B. pfeifferi transcripts) and subject (B. glabrata BB02 annotated proteins). Of these, 19 were GIMAPs. DE analysis of $B$. pfeifferi AIG genes (Additional file 5: Table S4) show that at 3-day post-exposure (dpe) to S. mansoni, 4 GIMAPs were significantly up-regulated (from 4.66- to 40.20-fold) and 1 GIMAP gene was significantly downregulated (2.32-fold). With the cutoff values for $\mathrm{DE}$ genes used in this study (PPDE $\geq 0.95$, and fold change $>2$ ), no AIG genes were detected to be significantly expressed at 1 dpe to $S$. mansoni or in shedding snails (Additional file 5: Table S4). Additionally, 15 B. glabrata AIG genes with clear B. pfeifferi homologs were identified in the organ expression study in Fig. 5.

\section{IV). Comparisons of expression levels of AIG gene family members in strains of B. glabrata susceptible ( $M$ line) or resistant (BS-90) to S. mansoni, before and after exposure to this parasite}

At constitutive levels (Table 1, Additional file 4: Table S3, Additional file 5: Table S4), for at least one of the three direct strain comparisons, 34 AIG genes were more highly expressed in schistosome-resistant BS-90 snails than in susceptible M line snails (19 GIMAPs, 11 AIG genes without coiled-coils and 4 partial AIG genes). Several AIG family members showing no expression in resting $M$ line snails were notable for being expressed at much higher resting levels in BS-90 snails. Among them, Bg17413 (GIMAP) and Bg34408 (AIG) have exceptionally higher average raw reads counts $(\sim 3000)$ compared to the rest 32 AIG genes $(\sim 300)$. Conversely, 21 AIG genes were over-expressed in $\mathrm{M}$ line relative to BS-90 snails (13 GIMAPs, 5 AIG without coiled-coils and 3 partial AIG genes).

Both B. glabrata strains showed relatively modest differences in AIG gene expression between unexposed and S. mansoni-exposed snails. For both strains the overall, but by no means exclusive trend, was towards down-regulation in snails exposed to S. mansoni. Bg7141 was notable for being upregulated in $M$ line snails shedding S. mansoni cercariae. Many (49) of the 111 AIG genes (including partial AIG genes) showed no transcriptional differences (fold change $\leq 2$ ) between strains, or within a strain following exposure to infection. GIMAPs comprised the majority (37 of 62) of all significantly expressed AIG genes in this study. Regarding special domains, only the Pkinase domain (Bg690) or dual-AIG1 domains were represented as significantly expressed among strain comparisons (Additional file 5: Table S4).

\section{Analysis of relationships among B. glabrata AIG genes}

Shown in Fig. 6a is a tree of 91 AIG genes with complete AIG1 domain sequences for B. glabrata (sequence alignments in Additional file 6: File S1). This tree revealed five different clades (I-V) of B. glabrata AIG1 domain sequences, with good bootstrap support values ( $>75 \%)$. There are two individual branches representing the genes Bg8770 and Bg9801 with slightly lower bootstrap support (both at $68 \%$ ).

In Fig. $6 \mathrm{~b}$, a schematic of each AIG gene is presented showing the different types of domains represented with different colors. The majority of the 91 AIG genes contain one AIG1 domain, but there are 8 AIG genes with dual AIG1 domains distributed in clades I, II and III. Both AIG1 domains were included within the gene tree analysis. When two AIG1 domains occur in the same gene, they tend to cluster within the same clade (for example, Bg32113_2 clusters with Bg32113_1 in clade I), although such pairs are not always each other's closest match. The other AIG genes containing special functional domains (death domains, protein kinase domain, hint/hedgehog domain, armadillo-type fold) were dispersed on the tree.

We noted AIG genes that lie close together in the genome and have similar domain architectures tend to cluster together on the tree. For instance, for one group of AIG genes in clade III (Fig. 6a), all are located in one tandem array (Fig. 4), and all are GIAMPs. Three genes in one sub-cluster of this clade (Bg9641, Bg39329, and Bg23073) had dual AIG1 domains as well as transmembrane domains and coiled-coil domains. By virtue of containing the largest tandem array of AIG genes (10 GIMAPs and 1 partial AIG) with close evolutionary relationships and by having dual-AIG1 domains, one particular scaffold region Scaffold 39:335311-543,941 seems to be a potential evolutionarily hot spot for generation of GIMAP genes through tandem gene duplication.

Gene structures of the 91 AIG genes were summarized with exon, intron and untranslated region (UTR) in different colors (Fig. 6c). Most of the gene structures show that one exon encodes one AIG1 domain and dual-AIG1 domains were encoded by two exons.

\section{AIG genes in 9 additional species of Mollusca}

The same workflow used to identify B. glabrata AIG genes was applied to 9 other molluscan species with well-annotated genomes (Table 2). Although the initial 
Table 1 Summary of AIG genes expression in B. glabrata BS-90 and M line at constitutive level and response to S. mansoni exposure

\begin{tabular}{|c|c|c|c|c|c|c|c|c|c|c|c|c|c|c|}
\hline \multirow{2}{*}{ Gene Id } & \multirow{2}{*}{$\begin{array}{l}\text { Brief ID } \\
\text { used in text }\end{array}$} & \multirow{2}{*}{ NCBI Annotation } & \multirow{2}{*}{ Type of AIG } & \multicolumn{3}{|c|}{ Constitutive comparsion } & \multicolumn{4}{|c|}{ BS-90 vs. S. mansoni } & \multicolumn{4}{|c|}{ M line vs. S. mansoni } \\
\hline & & & & $12 \mathrm{hrs}$ & 2d & 40d & $12 \mathrm{hrs}$ & 2d & 8d & $40 \mathrm{~d}$ & $12 \mathrm{hrs}$ & $2 d$ & $8 d$ & $40 d$ \\
\hline BGLB017413 & Bg17413 & GTPase IMAP family member 7 -like & GIMAP & 12.56 & 11.13 & 12.29 & ns & ns & ns & ns & ns & ns & ns & ns \\
\hline BGLB034427 & Bg34427 & GTPase IMAP family member 7 -like & GIMAP & 10.26 & 9.61 & 9.61 & ns & ns & ns & ns & ns & ns & ns & ns \\
\hline BGLB039682 & Bg39682 & GTPase IMAP family member 1-like, partial & Partial AIG & 9.91 & 9.49 & 3.20 & ns & ns & ns & ns & ns & ns & ns & ns \\
\hline BGLB013945 & Bg13945 & GTPase IMAP family member 4-like & AIG & 8.85 & 9.12 & ns & ns & ns & ns & ns & ns & ns & ns & ns \\
\hline BGLB034408 & Bg34408 & dentin sialophosphoprotein-like & AIG & 8.37 & 7.86 & 9.66 & ns & ns & ns & ns & ns & ns & ns & ns \\
\hline BGLB009801 & Bg9801 & putative protein PHLOEM PROTEIN 2-LIKE A3 & AIG & 8.36 & 6.48 & 7.88 & ns & ns & -2.61 & ns & ns & ns & ns & ns \\
\hline BGLB028209 & Bg28209 & putative protein PHLOEM PROTEIN 2-LIKE A3, partial & GIMAP & 6.81 & 6.76 & 6.58 & ns & ns & ns & ns & ns & ns & ns & ns \\
\hline BGLB004821 & Bg4821 & protein AIG1-like & GIMAP & 6.31 & 5.36 & 7.22 & ns & ns & ns & ns & ns & ns & ns & ns \\
\hline BGLB005730 & Bg5730 & GTPase IMAP family member 7 -like & GIMAP & 6.16 & 6.45 & ns & ns & ns & ns & ns & ns & ns & ns & ns \\
\hline BGLB039962 & Bg39962 & uncharacterized protein LOC106070566, partial & Partial AIG & 5.72 & 3.49 & 4.42 & ns & ns & ns & ns & ns & ns & ns & ns \\
\hline BGLB021204 & Bg21204 & protein AIG1-like & Partial AIG & 5.50 & 6.52 & 4.78 & ns & ns & ns & ns & ns & ns & ns & ns \\
\hline BGLB029877 & Bg29877 & GTPase IMAP family member 7 -like & GIMAP & 5.14 & 4.08 & 3.59 & -1.45 & ns & ns & ns & ns & ns & ns & ns \\
\hline BGLB032113 & Bg32113 & uncharacterized protein LOC106068839 & GIMAP & 4.26 & 6.09 & 3.61 & -1.96 & -1.06 & ns & ns & ns & ns & ns & ns \\
\hline BGLB016704 & Bg16704 & putative protein PHLOEM PROTEIN 2-LIKE A3 & GIMAP & 3.10 & 2.50 & ns & ns & ns & -1.93 & ns & ns & ns & ns & ns \\
\hline BGLB033631 & Bg33631 & GTPase IMAP family member 7 -like, partial & AIG & 3.03 & 2.90 & ns & -3.57 & ns & ns & ns & ns & ns & ns & ns \\
\hline BGLB009641 & Bg9641 & uncharacterized protein LOC106072015 & GIMAP & 2.37 & ns & ns & ns & ns & ns & ns & ns & ns & ns & ns \\
\hline BGLB023869 & Bg23869 & uncharacterized protein LOC106058609 & Partial AIG & 1.85 & ns & ns & ns & ns & ns & ns & ns & ns & ns & 1.75 \\
\hline BGLB010064 & Bg10064 & GTPase IMAP family member 7-like isoform X1 & GIMAP & 1.60 & 2.08 & 2.11 & ns & ns & ns & ns & ns & ns & ns & ns \\
\hline BGLB016943 & $\mathrm{Bg} 16943$ & GTPase IMAP family member 7-like isoform X1 & GIMAP & 1.46 & 1.58 & 1.43 & ns & ns & ns & ns & ns & ns & ns & ns \\
\hline BGLB031582 & Bg31582 & GTPase IMAP family member 7-like & GIMAP & 1.12 & ns & ns & ns & ns & ns & ns & ns & ns & 1.03 & ns \\
\hline BGLB029530 & Bg29530 & GTPase IMAP family member 8-like & AIG & ns & 8.16 & ns & ns & ns & -5.41 & ns & ns & ns & ns & ns \\
\hline BGLB034455 & Bg34455 & protein AIG1-like, partial & AIG & ns & 5.12 & 2.41 & ns & ns & ns & -1.70 & ns & ns & ns & ns \\
\hline BGLB023069 & Bg23069 & GTPase IMAP family member 7-like, partial & GIMAP & ns & 2.55 & ns & ns & ns & -1.44 & ns & ns & ns & ns & ns \\
\hline BGLB012262 & Bg12262 & GTPase IMAP family member 7 -like & GIMAP & ns & ns & 3.70 & ns & ns & ns & ns & ns & ns & ns & ns \\
\hline BGLB033791 & Bg33791 & uncharacterized protein LOC106071107 & AIG & ns & ns & 3.66 & ns & ns & ns & -2.01 & ns & ns & ns & ns \\
\hline BGLB035925 & Bg35925 & uncharacterized protein LOC 106053987 & GIMAP & ns & ns & 3.36 & ns & ns & ns & ns & ns & ns & ns & ns \\
\hline BGLB038034 & Bg38034 & GTPase IMAP family member 8-like & $\mathrm{A} \mid \mathrm{G}$ & ns & ns & 2.10 & ns & ns & ns & ns & ns & ns & ns & ns \\
\hline BGLB011835 & Bg11835 & putative protein PHLOEM PROTEIN 2-LIKE A3 & GIMAP & ns & ns & 1.77 & ns & ns & ns & ns & ns & ns & ns & ns \\
\hline BGLB006164 & Bg6164 & GTPase IMAP family member 7-like & AIG & ns & ns & 1.42 & ns & ns & ns & ns & ns & ns & ns & ns \\
\hline BGLB008944 & Bg8944 & protein AIG1-like & GIMAP & ns & ns & 1.37 & ns & ns & ns & -1.05 & ns & ns & ns & ns \\
\hline BGLB032825 & Bg32825 & protein AIG1-like, partial & GIMAP & ns & ns & 1.31 & -1.48 & ns & 1.48 & ns & ns & ns & ns & ns \\
\hline BGLB014138 & $\mathrm{Bg} 14138$ & putative protein PHLOEM PROTEIN 2-LIKE A3, partial & GIMAP & ns & ns & ns & ns & ns & ns & ns & -1.13 & ns & ns & ns \\
\hline BGLB008947 & Bg8947 & protein AIG1-like & GIMAP & ns & ns & ns & ns & ns & ns & ns & ns & ns & ns & -1.72 \\
\hline BGLB039543 & Bg39543 & protein AIG1-like & GIMAP & ns & ns & ns & ns & ns & -7.11 & ns & ns & ns & ns & ns \\
\hline BGLB037886 & Bg37886 & GTPase IMAP family member 4-like, partial & AIG & ns & ns & ns & ns & ns & -3.05 & ns & ns & ns & ns & ns \\
\hline BGLB025758 & Bg25758 & protein AIG1-like & Partial AIG & ns & ns & ns & 1.06 & ns & ns & ns & ns & ns & ns & ns \\
\hline BGLB034464 & Bg34464 & uncharacterized protein LOC106063447 & Partial AIG & ns & ns & -1.29 & ns & ns & ns & ns & ns & ns & ns & ns \\
\hline BGLB025549 & Bg25549 & GTPase IMAP family member 7-like & AIG & ns & ns & -2.00 & -3.22 & ns & ns & ns & ns & ns & ns & -1.24 \\
\hline BGLB004899 & Bg4899 & uncharacterized protein LOC 106060228 & GIMAP & ns & ns & -3.11 & -2.77 & -1.24 & -1.29 & ns & -2.01 & -1.85 & ns & ns \\
\hline BGLB027157 & Bg27157 & uncharacterized protein LOC106053184 & GIMAP & ns & ns & -3.87 & ns & ns & -2.45 & ns & ns & ns & ns & 1.10 \\
\hline BGLB009639 & Bg9639 & uncharacterized protein LOC106072017 isoform X2 & GIMAP & ns & -1.32 & ns & ns & ns & ns & ns & ns & ns & ns & ns \\
\hline BGLB029478 & Bg29478 & GTPase IMAP family member 8-like & GIMAP & ns & -1.36 & ns & ns & ns & ns & ns & ns & ns & ns & ns \\
\hline BGLB011834 & Bg11834 & GTPase IMAP family member 6 -like & GIMAP & ns & -4.55 & ns & ns & ns & ns & ns & ns & ns & ns & ns \\
\hline BGLB038543 & Bg38543 & GTPase IMAP family member 7-like & GIMAP & ns & -1.05 & ns & ns & ns & ns & ns & ns & ns & ns & ns \\
\hline BGLB032430 & Bg32430 & uncharacterized protein LOC 106050154 & AIG & ns & -1.11 & ns & ns & ns & ns & ns & ns & ns & ns & ns \\
\hline BGLB028483 & Bg28483 & protein AIG1-like, partial & AIG & ns & -1.72 & -3.65 & ns & ns & ns & ns & ns & ns & ns & ns \\
\hline BGLB024203 & Bg24203 & uncharacterized protein LOC 106075341 & Partial AIG & ns & -1.16 & ns & ns & ns & ns & ns & ns & ns & ns & ns \\
\hline BGLB017488 & Bg17488 & uncharacterized protein LOC106050236 isoform X1 & GIMAP & -1.04 & ns & -1.83 & ns & ns & -1.22 & ns & ns & ns & ns & ns \\
\hline BGLB033169 & Bg33169 & uncharacterized protein LOC 106072012 & Partial AIG & -1.17 & ns & -2.58 & ns & ns & ns & ns & ns & ns & ns & ns \\
\hline BGLB008269 & Bg8269 & putative protein PHLOEM PROTEIN 2-LIKE A3 & GIMAP & -1.46 & ns & ns & ns & ns & ns & ns & ns & ns & ns & ns \\
\hline BGLB000679 & Bg679 & GTPase IMAP family member 7-like & GIMAP & -1.56 & -1.58 & ns & ns & ns & ns & ns & ns & ns & ns & ns \\
\hline BGLB028741 & Bg28741 & protein AIG1-like, partial & AIG & -1.63 & -1.57 & ns & -1.31 & ns & ns & ns & -1.24 & ns & ns & ns \\
\hline BGLB038793 & Bg38793 & uncharacterized protein LOC106059809 & GIMAP & -2.20 & -2.93 & ns & ns & ns & ns & ns & ns & ns & ns & ns \\
\hline BGLB024015 & Bg24015 & uncharacterized protein LOC 106058610 & GIMAP & -2.32 & -3.00 & -1.80 & ns & ns & 1.40 & ns & ns & ns & ns & ns \\
\hline BGLB000690 & Bg690 & rho-associated protein kinase let-502-like & GIMAP & -2.54 & -3.14 & ns & ns & ns & ns & ns & ns & ns & ns & ns \\
\hline BGLB039329 & Bg39329 & uncharacterized protein LOC106072011 & GIMAP & -2.83 & ns & -2.66 & ns & ns & ns & ns & ns & ns & ns & ns \\
\hline BGLB026189 & Bg26189 & GTPase IMAP family member 4-like, partial & AIG & -5.03 & ns & -5.30 & ns & ns & ns & ns & ns & ns & ns & ns \\
\hline
\end{tabular}

Notes

1. Value in cells represents the value of log2(fold change) of the gene. Any up-regualted DE gene with log2(fold change) greater than 1 is highlighted in red, and any down-regualted DE gene with $\log 2$ (fold change) less than -1 is highlighted in green

2. The $\mathbf{1 2} \mathbf{~ h , ~} \mathbf{2 d}, \mathbf{8 d}$ and $\mathbf{4 0 d}$ represent the 4 sampling time points, which are time-matched unexposed controls and exposed snails for comparison

3. ns: no significant expression difference detected. Gene with PPDE $>0.95$ and fold change $>2$ is considered to be differentially expressed in this study

Shown are 3 comparisons of constitutive levels of AIG gene expression in BS-90 snails relative to M line snails (all snails were unexposed time-matched controls, with those at 0.5 and 2 dpe representing juvenile snails and those at 40 dpe being adult snails). Also, for both M line and BS-90 snails, there are 4 separate within strain comparisons of the effects of S. mansoni exposure (for $0.5,2,8$ and 40 dpe) on AIG gene expression. Note that by 40 dpe, exposed BS-90 snails contain little or no S. mansoni DNA, and exposed $\mathrm{M}$ line snails were shedding cercariae 
a

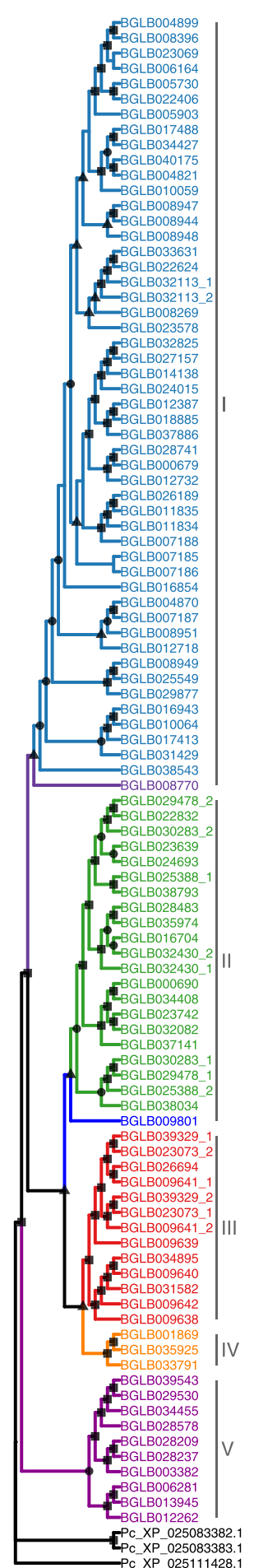

b
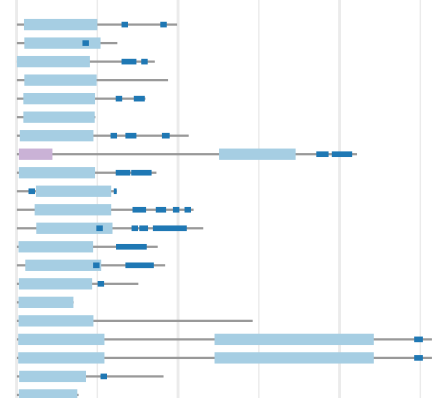

2

$-=$

-

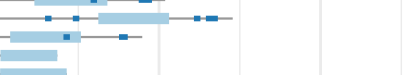

r.

$\longrightarrow+$

$\square$

$=$

-

$=-$

$=$

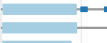

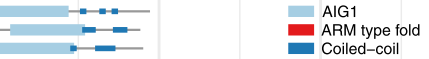

Coiled-coil
DD-CARD

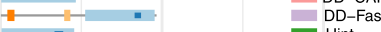

$=-\left[\begin{array}{l}\text { Hint } \\ \text { Pkinase }\end{array}\right.$
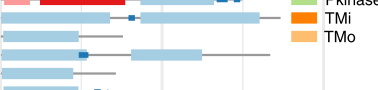

$-$

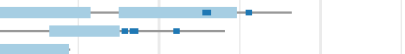

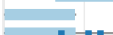

$+\infty$

$+$

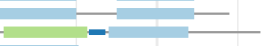

(n)

2
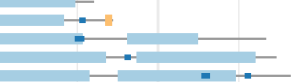

$+1-2$

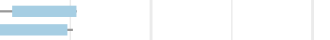

$\square \div-\square$
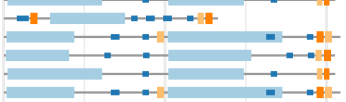

$+=-$

$\rightarrow-1$

$\longrightarrow$
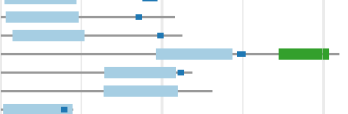

$=$

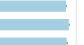

$-1$

-

$+$

$500 \quad 1000$

Protein Domain Structure (aa)
C

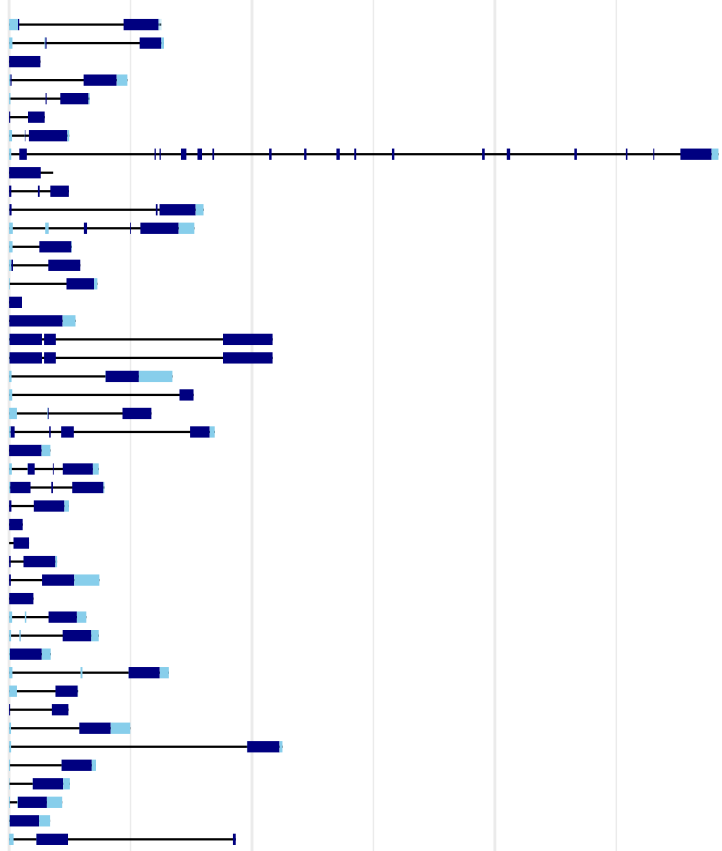

Gene Structure

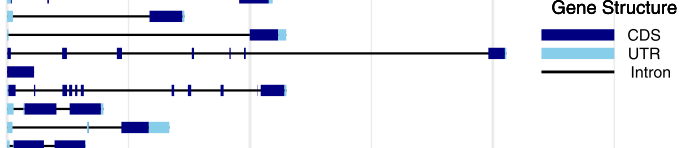

담

$+m$

-

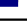
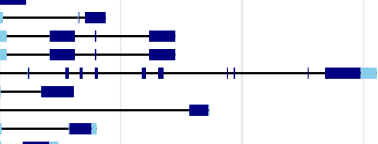

$-1$

-

$\longrightarrow$

-

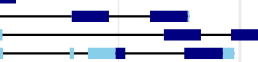

$\longrightarrow \square$
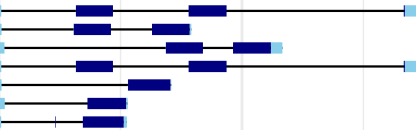

$\longrightarrow$

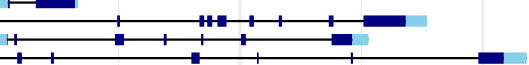

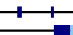

프.

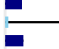

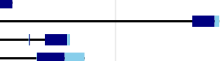

픔

?
0
1000020000

Gene Exon/Intron Structure (nt)

Fig. 6 (See legend on next page.) 
(See figure on previous page.)

Fig. 6 Relationships, domain architecture and gene structure of AIG genes in B. glabrata. a. A maximum likelihood (ML) phylogenetic tree of AIG1 domain sequences, with bootstrap values of 50 and greater noted with black shapes on the nodes (square 90-100, circle 70-89, triangle 50-69). For those AIG genes with dual-AIG1 domains, labels of "_1" and "_2" were added to the corresponding gene ID to differentiate the first and the second AIG1 domains. The tree topology revealed five clades (I-V) with well-supported bootstrap values (> 75), with two separate individual branches appearing among the five clades. b. Domain architectures of representative protein sequence of each AIG gene are listed. The featured domains include AIG1, coiled-coil, TMi (transmembrane domain from inside to outside of a membrane), TMo (transmembrane domain from outside to inside of a membrane), and other special functional domains: DD-CARD (death domain CARD type), DD-Fas (death domain Fas type), Hint (hint/hedgehog), armadillo (ARM)-type fold and Pkinase (protein kinase). c. Gene structures corresponding to representative transcripts of AIG genes were shown with exon, intron and untranslated region (UTR) in different colors

AIG1 domain search with the HMM profile revealed some possible relevant hits, the follow up InterProScan analysis showed that some of these candidates actually belonged to other GTPase families with the same superfamily P-loop NTPase structure. For example, initially, 20 putative candidate genes were discovered with the HMM profile in Octopus bimaculoides and 17 in Euprymna scolopes, however none were confirmed as bonafide AIG genes after screening by InterProScan. Here, only those fitting the AIG gene criteria of this paper were summarized in Table 2.

A maximum likelihood tree (Fig. 7, Additional file 6: File S1 for alignments) was constructed using complete AIG1 sequences from the 8 molluscan species with AIG genes from the coral Acropora millepora as outgroup. It is noteworthy that coral, gastropod and bivalve sequences were for the most part segregated from one another in the tree. One exception was the basal patellogastropod gastropod Lottia gigantea which had an isolated branch clustering within a number of sequences from the bivalve Mizuhopectin, and a small group of sequences clustering with another group of Mizuhopectin sequences (a small blue cluster at the bottom left, Fig. 7). Also noteworthy in this regard was Pomacea for which AIG1 domain sequences were diverse. Most arose from a single basal sequence distinct from the one basal sequence for the entire heterobranch group. However, a small subgroup of Pomacea canaliculata was also noticed embedded within a group otherwise composed of bivalve sequences. Interestingly, all members of this unusual subgroup are from the second, C-terminal AIG1 domain in dual-AIG1 domain containing genes. Heterobranch sequences were also diverse and showed evidence of distinct expansions among Aplysia, Elysia and Biomphalaria. In general, lineage specific expansions are observed as a major trend of molluscan AIG gene diversification with at least 3 such expansions noted for Elysia, 4 for Aplysia and 6 for Biomphalaria. Similar expansions are noted for the bivalves Mizuhopectin and the two Crassostrea species. As many as three lineage expansions can be traced back to a common basal state.

We also examined AIG genes in a second prominent Biomphalaria species, the widespread African schistosome vector species $B$. pfeifferi. Although the whole genome of $B$. pfeifferi is not available, assembled transcriptome sequences generated from a previous RNA-Seq study were used [25]. We followed the same methods of AIG gene identification and gene tree construction (Fig. 8). The relatively close degree of relationship between the two taxa shown in this figure relative to Fig. 7 is revealed by the extent to which the AIG1 domain sequences of B. glabrata and B. pfeifferi are intermingled throughout the tree. Although a couple of early diverging single branches for B. glabrata were noted, the remaining groups initiated from deeper branches which

Table 2 Summary of predicted numbers of AIG family members in B. glabrata and 9 molluscan species

\begin{tabular}{|c|c|c|c|c|c|}
\hline Organism & Common Name & AIG & Partial AIG & Total & GIMAP \\
\hline Biomphalaria glabrata & Freshwater snail & 91 & 20 & 111 & 64 \\
\hline Elysia chlorotica & Eastern emerald elysia & 18 & 3 & 21 & 14 \\
\hline Aplysia californica & California sea hare & 28 & 2 & 30 & 17 \\
\hline Pomacea canaliculata & Golden apple snail & 89 & 8 & 97 & 41 \\
\hline Lottia gigantea & Owl limpet & 11 & 12 & 23 & 2 \\
\hline Mizuhopecten yessoensis & Yesso scallop & 28 & 2 & 30 & 20 \\
\hline Crassostrea gigas & Pacific oyster & 37 & 2 & 39 & 25 \\
\hline Crassostrea virginica & Eastern oyster & 76 & 2 & 78 & 57 \\
\hline Octopus bimaculoides & California two-spot octopus & 0 & 0 & 0 & 0 \\
\hline Euprymna scolopes & Hawaiian bobtail squid & 0 & 0 & 0 & 0 \\
\hline
\end{tabular}

Phylogenetic relationships among B. glabrata and 9 additional molluscan species were inferred according to published literature [37-39] 


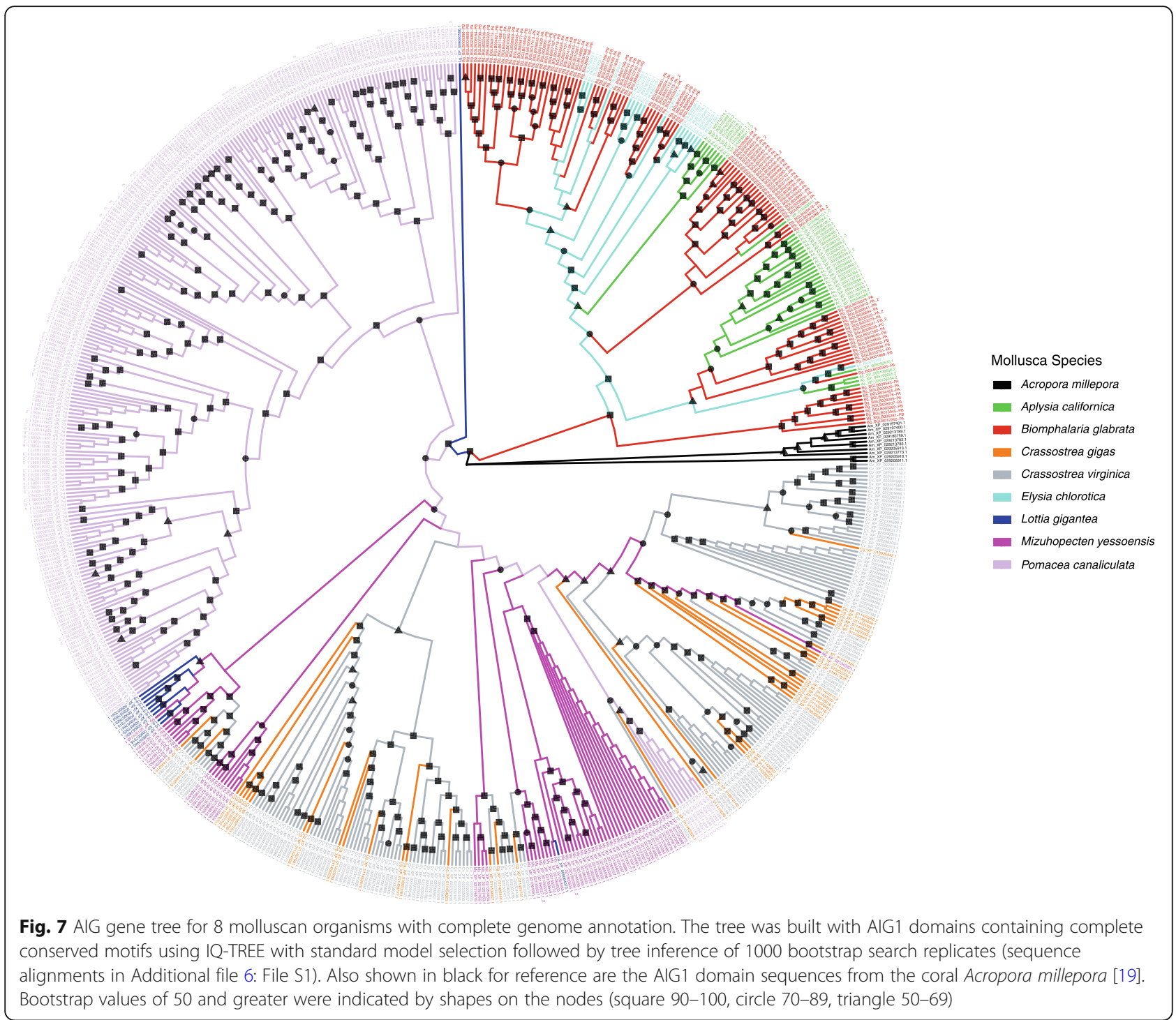

seemed to originate with $B$. pfeifferi sequences, but most contained terminal branches from both species, again emphasizing the overall similar representation of AIGs across the two species. At least five major clusters contained only $B$. pfeifferi sequences, and the overall number of terminal branches represented by $B$. pfeifferi was higher (142) than for B. glabrata (101). As a comparison, an oyster AIG gene tree for the congeners $C$. gigas and C. virginica (Fig. 8, right) was also generated showing a clade distribution consistent with that seen in Fig. 7. More multi-member clades with species specific expansion were shown on the Crassostrea tree as compared with the Biomphalaria tree (Fig. 8, left).

\section{Discussion}

The AIG gene family (containing GIMAPs) is recognized for its role in plants in defense from bacterial infection, and in mammals where GIMAPs play a role in regulation and maintenance of lymphocyte numbers and in phagolysosomal processing. GIMAPs are transcribed following injection of freshwater snail Biomphalaria glabrata with PAMPs like LPS [24]. Here for the first time we characterize the AIG gene family in B. glabrata which is an important vector for the widespread parasite of humans, Schistosoma mansoni. We also provide additional AIG family information for the related schistosome vector species, B. pfeifferi. We begin the exploration of their functional roles by documenting their expression patterns in various contexts including in B. glabrata strains susceptible or resistant to $S$. mansoni, and seek to explain origins and diversification of AIG genes of B. glabrata by comparison with those of other molluscs.

We first identified the essential motifs associated with the AIG1 domain in several B. glabrata AIG genes. Conserved motifs G1-G3, CB (conserved box), G4, G5/IAN were observed to have kept a consistent order and retained recognizable consensus sequences. We also observed 

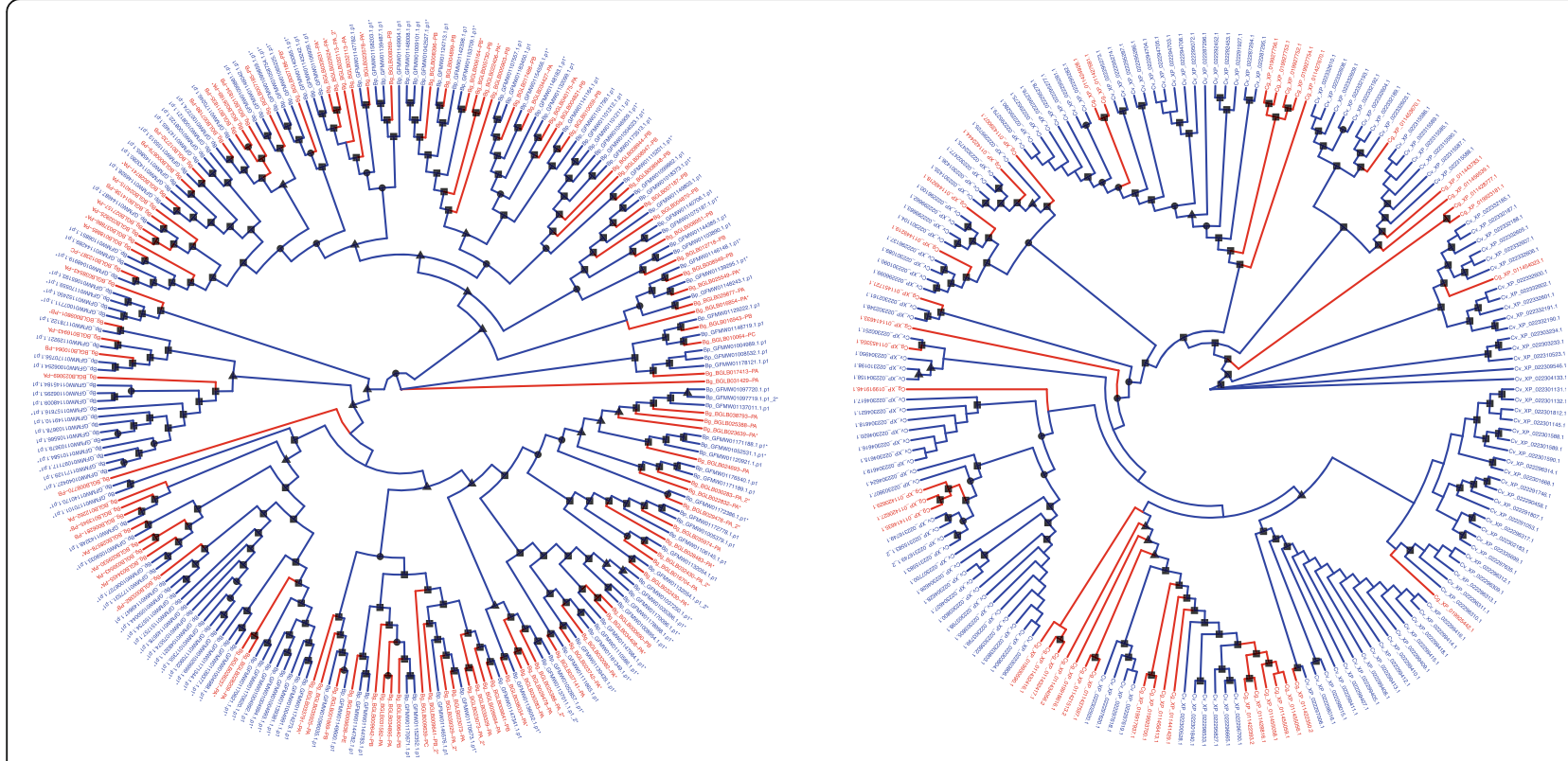

Biomphalaria spp. B. glabrata $=$ B. pfeifferi

Crassostrea spp. $=$ C. gigas $\square$ C. virginica

Fig. 8 AIG gene tree for congeners B. glabrata and B. pfeifferi or C. gigas and C. virginica. The phylogenetic tree was built with AIG1 domains containing complete conserved motifs, using IQ-TREE with standard model selection followed by tree inference of 1000 bootstrap search replicates (sequence alignments in Additional file 6: File S1). Bootstrap values of 50 and greater were indicated by shapes on the nodes (square 90-100, circle 70-89, triangle 50-69)

distinct sequence substitutions within motifs unique to different species, including for B. glabrata. This is one potential indication of an ancient origin of the AIG1 domain, followed by substantial modifications in those groups of animals that have maintained the domain.

A total of 111 genes with complete or partial AIG1 domains were found in B. glabrata, of which 91 had complete AIG1 domains. The 91 genes with complete AIG1 domains were diverse with respect to the additional domains they contained, with 19 domain arrangements noted, five of which had two separated AIG1 domains. The functionality of B. glabrata AIG genes is potentially expanded by recruiting coiled-coils, transmembrane domains and other domains with specific functions. A multimerization option favored by the presence of coiled-coil domains may lead to further functional options involving larger macromolecular complexes [34]. The lack of signal peptides and the presence of transmembrane domains frequently found in $B$. glabrata AIGs are indicative of membrane-associated activities. Other specialized domains associated with the $B$. glabrata AIG1 domain include death domain superfamily members [40, 41], caspase activation and recruitment (CARD) domains [42, 43], and a protein kinase domain for a B. glabrata GIMAP (the latter also known from Atlantic salmon (Salmo salar) or fungi). Also found were Armadillo-type fold domains possibly facilitating large molecule (protein or nucleic acids) binding capability [44, 45] and a Hint domain associated with proteolysis functions [46].

An analysis of the distribution of AIG genes among the scaffolds of the BB02 B. glabrata genome shows that 50 AIG footprints (31 B. glabrata GIMAPs, 9 AIG genes without coiled-coils, and 10 partial AIG genes) were found to be tandemly arrayed in clusters ranging from 2 up to 11 genes. AIG genes with similar genomic location and functional features tend to cluster together. Although it is hard to reconstruct the mechanisms involved in AIG gene family expansions, the presence of AIG clusters and of occasional dual AIG1 domains in B. glabrata suggest segmental duplication as the main mechanism of family expansion. Clusters of tandemly arrayed AIG genes have also been found in humans and plants. Fusing of one open reading frame to another helps maintain a consistent ratio of transcription and translation for the co-occurring functional domains. Scaffold 39:335311-543,941, containing 10 evolutionarily similar GIMAPs in tandem array, seems like a potential hot spot for generation of GIMAP genes via tandem gene duplication. Tandem duplications (or equivalently unequal crossovers) have been found to account for $14 \%$ of all genes in 11 vertebrate genomes [47]. The high degree of homology seen in tandem arrays facilitates both unequal recombination and gene conversion 
which further reinforces homology resulting in large gene families [48]. Multiple rounds of unequal crossovers may lead to increases or decreases in the copy number of AIG genes, making it more flexible in response to changing environmental challenges [47, 48].

Towards a better functional understanding of AIG genes, we examined their expression in four different contexts (Additional file 5: Table S4). First, we profiled AIG genes expression in 12 different organs from unstimulated BB02 B. glabrata. We found over half of the AIG genes displayed considerable differences in organspecific expression profiles, with some organs like the terminal genitalia and albumen gland having AIG gene expression patterns more similar to one another. More than $80 \%(47 / 58)$ of the AIG genes we noted to be expressed in the sampled organs are GIMAPs. Some groups of transcripts with similar patterns of expression were also noted. There is also evidence that these groups represent either related genes, or genes found within the same tandem array. For example, in the tandem array on the scaffold 39, two loci (Bg26694 and Bg9640) were both overexpressed in digestive gland, and three loci (Bg9642, Bg31582 and Bg34895) were all under expressed in salivary glands.

Second, we reexamined the expression results from the microarray study of schistosome resistant BS-90 B. glabrata injected with various PAMPs, including LPS [24]. Four AIG genes, two of which were GIMAPs, were among the most highly expressed genes recovered (up to 13-fold), strongly suggestive of a role for AIGs in response to bacterial PAMPs. Third, we also analyzed an extensive RNA Seq transcriptomics data for field-derived Kenyan Biomphalaria pfeifferi exposed for 1,3 , or $30+$ days to $S$. mansoni, relative to unexposed controls [25]. Of 28 homologs for B. glabrata AIG genes identified in the B. pfeifferi dataset, four GIMAPs were highly expressed in schistosome-exposed snails at 3 days post-exposure, relative to unexposed control snails. Although the responses were not as dramatic as noted following injection of LPS, they nonetheless indicate snails normally highly susceptible to $S$. mansoni infection can mount an AIG response involving multiple genes.

Fourth, an RNA Seq transcriptomics study revealed that several AIG family members had much higher constitutive levels of expression $(10+$ fold $)$ in the $S$. mansoni-resistant BS-90 snails relative to susceptible $\mathrm{M}$ line snails. Although neither snail strain showed particularly noteworthy or consistent changes in AIG gene expression following exposure to $S$. mansoni infection, the strikingly high constitutive expression shown by several AIG genes in BS-90 snails makes them excellent candidates as resistance-associated genes. One of these genes, Bg17413, a GIMAP gene (Additional file 5: Table S4), was significantly upregulated in both $B$. glabrata exposed to LPS and
PGN [24] and in B. pfeifferi snails at 3-day post exposure to $S$. mansoni [25].

Collectively the transcription studies reveal that AIG family members have complex patterns of expression in different organs, can respond strongly to bacterial PAMPs, and show remarkably high resting levels of expression in snails resistant to schistosomes. Further studies assessing the impact of knock-down of single or multiple highly expressed AIG genes on the resistance of BS-90 snails to S. mansoni should be undertaken. BG7141, a GIMAP highly upregulated in M line snails with full-blown patent infections of $S$. mansoni should also be investigated as a possible growth factor for S. mansoni or as part of a response to protect the snail from consequences of infection. Other AIG genes, such as those responding most dramatically to PAMP exposure (Bg11834, Bg7188, Bg9640, etc.) [24] also deserve further consideration as key factors in the defense responses to microbial pathogens.

One of the most interesting aspects of AIG genes is their unusual phylogenetic distribution, with molluscs being one of the few groups of lophotrochozoans to possess them. Although no AIG genes were found within the Octopus bimaculoides and Euprymna scolopes (squid) genomes, more representatives need to be examined to assess presence or absence of AIG genes among cephalopods. The five gastropods and three bivalve genomes available reveal different levels of AIG gene expansion, ranging from 23 to 111 (Table 2). Of the $91 \mathrm{~B}$. glabrata AIG genes with complete AIG1 domain, 64 were identified as GIMAPs, with both of these totals being higher than for any of the other nine mollusc species examined. With the exception of Danio (zebrafish), B. glabrata has more GIMAP loci than any other species thus far investigated.

The AIG gene tree is complex and does not simply recapitulate phylogenetic relationships among the taxa represented. Nonetheless, some phylogenetic signals are provided in the tree. For instance, the AIG genes for three relatively closely related heterobranch gastropods (A. californica, $E$. chlorotica and B. glabrata) are grouped together from a single major branch. The terminal branches stemming from this major branch show a remarkable intercalation of expanded subgroups of AIG genes representing all three species. Ten separate subgroups are seen for B. glabrata, whereas both $A$. californica and E. chlorotica have five. The more phylogenetically distant coenogastropod Pomacea canaliculata exhibits a separate expansion of AIG genes stemming from one major branch. How this picture would change with the addition of sequences from other coenogastropod species will prove to be most interesting. AIG gene sequences from the most basal gastropod, L. gigantea, represented on the tree occupy two major branches, one unique to L. gigantea but not expanded and nesting between the two gastropod branches identified above, and the second nested within a major branch that is otherwise mostly 
populated by bivalve AIG sequences. This second major branch is once again noteworthy for having smaller intercalated subgroup expansions representing all three bivalve species as well as $L$. gigantea. The extent to which the congeners C. gigas and C. virginica differ with respect to their AIG gene repertoires is noteworthy. This is quite in contrast to the differences in AIG gene repertoires noted between B. glabrata and B. pfeifferi in Fig. 8, for which differences are manifested mostly on the terminal branches on the tree. This implies that following a basic expansion in an ancestral species, there has since been only minor modifications as might be expected for two related species estimated to have diverged within the last 5 million years [49]. Additional sampling from more taxa will be required before we can fully interpret the patterns exhibited by the diverse repertoires of AIG genes among molluscs.

Given the status of current data and tool availability, there are caveats and limitation to our study. First, accuracy of AIG gene predictions is limited by completeness and accuracy of the reference genome and its annotation. AIG genes may be underestimated because some scaffolds do not cover a complete gene. Multi-domain AIG genes may have been overlooked if their component domains were on different scaffolds. Similar issues arise with transcriptome assembly if AIG expression is limited to particular organs and conditions. Genes not identified within a transcriptome are not necessarily absent from, or always silent within, the genome. Similarly, genome annotation is limited by our current knowledge of gene models and RNA-Seq/EST data. Second, our study used as a starting point the nine annotated molluscan genomes, so it skipped the steps involved in the initial annotation process. Gene annotation efforts may not always be able to fully distinguish between protein coding, or non-coding genes or pseudogenes. Further wet lab molecular techniques will be required for the final confirmation of existence and functionality of the AIG genes identified here. Third, the HMM profile/models used to scan signatures were built from protein databases containing large number of sequences from model organisms, so are likely to be somewhat biased against reliable domain identification in non-model organisms. This creates some uncertainty with respect to novel domain discovery. For example, some of the B. glabrata AIG genes contain long lengths of protein-encoding sequences with no known function. As BLAST searches of such sequences returned nothing from the NCBI nonredundant protein database, they may represent completely novel stretches of functional sequence, or reflect an assembly or annotation error. Fourth, given the complexity of the coiled-coil domain families including some subfamilies that are structurally conserved but have divergent sequences, AIG genes currently categorized as lacking coiled-coils may require reconsideration as bonafide GIMAPs as new prediction tools for coiled-coil domains are developed.

\section{Conclusions}

A systematic investigation of the B. glabrata genome has revealed the presence of 91 AIG genes with complete AIG1 domains, more than known for any other organism except zebrafish. Most (64) AIG family members in $B$. glabrata are GIMAPs. The B. glabrata AIG1 domain appears in several novel combinations with other domains suggestive of a variety of possible functions. Several tandemly-arrayed AIG gene clusters appear in the B. glabrata genome, consistent with a process of segmental gene duplication in their origin. Some B. glabrata GIMAPs are strongly upregulated following exposure to bacteria or to S. mansoni indicative of a role in immunity. A role in interactions with $S$. mansoni is further suggested by the high constitutive levels of expression shown by several AIG genes from schistosome-resistant BS-90 snails as compared to susceptible $\mathrm{M}$ line snails. With respect to their distribution among molluscs, AIG genes are found in the available gastropod and bivalve genomes but thus far appear to be absent in cephalopods. The B. glabrata AIG genes localize within one major group on the AIG gene tree that includes only AIG genes from other heterobranch gastropods, but subgroups of B. glabrata AIGs are intercalated among the sequences of these other heterobranchs suggesting they have undergone several separate amplifications specific to B. glabrata. The curious pattern of apparent ancient presence followed by patterns of apparent loss in some lineages and expansions in others presents a fascinating puzzle awaiting further clarification. The approach taken here will help provide a framework for systematic experimental characterization of new biochemical and biological functions of AIG or GIMAP genes using modern tools like CRISPR/Cas knockout technology [50]. More specifically, we seek to learn how different species and strains of Biomphalaria compare with respect to how their AIG genes function in response to infection with the medically relevant parasite, S. mansoni.

\section{Methods}

\section{Data retrieval}

Protein and coding regions of gene sequences (CDS) and genome annotation features of B. glabrata BB02 strain, BglaB1.6 [36] were downloaded from VectorBase (https:// vectorbase.org/organisms/biomphalaria-glabrata/bb02/bgl ab16) [51]. Nine available and well-annotated molluscan genomes were downloaded from NCBI RefSeq [52] and GenBank [53]: the bivalves Crassostrea gigas (GCA 000297895.1), Crassostrea virginica (GCA_002022765.4), and Mizuhopecten yessoensis (GCA_002113885.2); the gastropods Aplysia californica (GCA_000002075.2), Elysia chlorotica (GCA_003991915.1), Lottia gigantea (GCA_ 
000327385.1), Pomacea canaliculata (GCA_003073045.1); and the cephalopod Octopus bimaculoides (GCA_001194 135.1). The genome and annotation for Hawaiian bobtail squid Euprymna scolopes which became available during the course of this study were obtained from CephRes Genomes Database (http://www.cephalopodresearch.org/ ceph_gdatab/).

\section{Computational identification of an AIG gene family in $B$. glabrata}

The characteristic AIG1 domain was used for computational analyses in the B. glabrata and the nine related molluscan genomes. Hmmsearch was performed to search for the AIG1 domain with the HMM model of AIG1 PF04548 from the Pfam database v32.0 [54]. InterProScan 5.34-73.0 software [55] was used to further confirm the AIG1 domain and scan signatures for other functional domains in the AIG1 domain-containing protein sequences of each species. Conservative motifs including the G1-G4, G5/ IAN motifs and a conserved box (CB) of the AIG1 domain were scanned within the CDD database [56] using CD-Search tools [57]. Additional features of AIG protein transmembrane domains and topology were predicted with TMHMM 2.0 [58], and coiled-coils were predicted by Coils [59] and their oligomeric state was predicted using LOGICOIL [60].

\section{Organ specific expression analysis of AIG genes in $B$. glabrata BB02 strain}

RNA sequencing reads of 12 organs from B. glabrata BB02 strain [36] were downloaded from NCBI Sequence Read Archive (SRA) with SRA run accession numbers SRR1509459- SRR1509470, and SRR1509473. The prefetch and fastq-dump command in the SRA Toolkit (http://ncbi.github.io/sra-tools/ and the SRA Toolkit Development Team) fastq files were used to download SRA data from NCBI, and then to extract fastq format sequences. The raw reads of each tissue were trimmed based on the base calling quality and read length using Trimmomatic v0.36 [61]. Clean reads were mapped to the reference B. glabrata BB02 genome using STAR v2.2.1 [62]. Gene counts measured in reads per kilobase of exon per million reads (RPKM) values were calculated using StringTie v1.3.5 [63]. Heatmap figures were generated using the $\mathrm{R}$ package pheatmap 1.0.12 [64].

\section{AIG homolog search and expression analysis of two previous studies on Biomphalaria spp}

From the microarray study of expression of BS-90 strain $B$. glabrata exposed to bacterial and schistosome-like PAMPs [24], all probes listed in the differential expressed genes were selected. Probe sequences were searched against cDNA sequences of BB02 B. glabrata using BLASTn with at least $>95 \%$ match identity. In the RNA-Seq study on Biomphalaria pfeifferi snails exposed to Schistosoma mansoni [25], homologs of AIGs from B. pfeifferi were identified through searching assembled transcriptome sequences by using BLASTp with cutoffs of identity $(>70 \%)$, query coverage $(>90 \%)$ and subject coverage (>90\%). For both of these searches, the corresponding gene IDs of AIG homologs for $\mathrm{BB} 02 \mathrm{~B}$. glabrata in Vectorbase were identified for further comparison analysis.

\section{Transcriptomics study on AIG genes in schistosoma susceptible- and resistant-strains of $B$. glabrata}

To further investigate AIG genes expression in B. glabrata, a comprehensive transcriptomics study of two $B$. glabrata strains (BS-90 snails resistant to S. mansoni, and $M$ line snails susceptible to this parasite) was carried out using next generation sequencing. Schistosoma mansoni (PR-1 strain) and the two strains of B. glabrata were maintained at Biology Department of the University of New Mexico as previously described [65, 66]. Juvenile snails (5-8 mm diameter) of both BS-90 and $\mathrm{M}$ line were individually put in the wells of 24-well plates, in $2 \mathrm{ml}$ artificial spring water (ASW) and exposed to $\sim 20 \mathrm{~S}$. mansoni miracidia per snail, for $6 \mathrm{~h}$. Control snails for each strain were treated similarly but were not exposed to S. mansoni miracidia, and represent the snails used to establish constitutive (resting) levels of AIG gene expression.

Snails of each group (Additional file 4: Table S3) were moved to aerated aquaria containing ASW at $25-27^{\circ} \mathrm{C}$ and fed with lettuce. Both $S$. mansoni-exposed snails and time-matched unexposed control snails of both strains were taken at $0.5,2$, and 40 days post-exposure (dpe); note that the same unexposed snails were used as controls for both the 2- and 8 dpe exposed snails because snails of these groups did not differ in size. These times were chosen to match key stages of $S$. mansoni development in the snail: 0.5 days, early penetration; 2 days, mother sporocyst establishment; 8 days, daughter sporocyst production; 40 days, full-fledged infection with production (shedding) of cercariae. Snails sampled at 0.5, 2, and 8 dpe were juveniles but snails collected at 40 dpe were adults because unexposed control snails produced eggs ( $S$. mansoni-infected snails are typically castrated by infection). For each strain and sampling time, 7-9 snails were collected. At $40 \mathrm{dpe}$, all remaining snails of both strains were isolated and checked for S. mansoni cercariae shedding. For this purpose, snails were placed individually in wells of 12-well plates in $2 \mathrm{~mL}$ ASW and held under artificial light for $2 \mathrm{~h}$. As expected, no resistant BS-90 snails shed cercariae. All snails were then allowed to recover in aquaria for 1-2 days prior to sampling. Snails sampled for this study were individually preserved in a $1.7 \mathrm{ml}$ tube containing $1000 \mu \mathrm{l}$ of TRIzol ${ }^{\mathrm{Tw}}$ reagent (Invitrogen) and stored at $-80^{\circ} \mathrm{C}$ until extraction. 
The RNA and DNA extraction of individual snail samples followed TRIzol ${ }^{\mathrm{Tm}}$ manufacturer's instructions (Invitrogen). PCR with $S$. mansoni specific primers aimed to the ND5 gene of S. mansoni [67] was used to verify the $S$. mansoni infection status for each schistosome-exposed snail. Only those snails exposed to $S$. mansoni and confirmed with PCR to be positive were considered to be "exposed" snails for this study. RNA samples were further purified using PureLink RNA Mini Kit (Thermo Fisher Scientific) to yield high-quality RNA. Quality and quantity of RNA extracted from each sample were measured with a Nanodrop 2000c spectrophotometer (Thermo Fisher Scientific) and Agilent 2100 Bioanalyzer (Agilent RNA 6000 Pico kit), respectively. RNA samples were stored at $-80^{\circ} \mathrm{C}$ and were used for library preparation within one week.

According to the sampling scheme in Additional file 4: Table S3, 3 6 replicates per group per sampling time point were selected for library preparation. Complementary DNA (cDNA) synthesis and Illumina NextSeq 500 sequencing was performed at the Molecular Biology Facility, Biology Department, the University of New Mexico. Synthesis of cDNA from each sample and library preparation followed the KAPA mRNA HyperPrep Kit Illumina ${ }^{\circ}$ Platforms protocol (Roche). Complementary DNA libraries were paired-end sequenced $(2 \times 150$ base reads $)$ on an Illumina NextSeq 500 instrument (Illumina).

\section{Differential expression (DE) analysis of the transcriptomics study}

For both M line and BS-90 strains, Illumina RNA Seq output for unexposed time-matched controls and PCRverified $S$. mansoni-exposed snails including raw reads with a score above 20 and length of at least $36 \mathrm{nt}$, were mapped to the reference BB02 B. glabrata genome [36] (Additional file 4: Table S3). Six groups of unexposed snails and 8 groups of $S$. mansoni-exposed snails were considered for various comparisons, including constitutive level comparisons between BS-90 and $\mathrm{M}$ line strains. The raw sequence data are available at NCBI under SRA accession: PRJNA591872.

All raw reads from Illumina sequencing were downloaded to local servers for bioinformatics analysis. Raw reads were trimmed and filtered using Trimmomatic v0.36 [61] with slide window of $4 \mathrm{nt}$, average score above 20 and minimum length of $36 \mathrm{nt}$. Filtered high quality reads were sorted based on the sample-specific adapters and mapped to the annotation updated Biomphalaria glabrata BB02 strain genome [36] from VectorBase [51], using STAR 2.5.3a [62]. Gene expression levels were estimated using software featureCounts [68]. Differential gene expression (DE) analysis was performed by using EBSeq v1.22.0 [69] with normalized clean reads, and DE analysis results were organized using SARTools 1.6.6 [70]. Workflow of read trimming and mapping was build using Unix shell commands with application GNU-Parallel [71] to perform jobs in parallel. A posterior probability of differential expression (PPDE) $\geq 0.95$ for EBSeq was set as cutoff for $\mathrm{DE}$ analysis. Due to the large number of $\mathrm{DE}$ genes discovered, only those DE genes with fold change (FC) value greater than $2(\mathrm{FC}>2)$ in either up- or downregulated were taken into further analyses. For constitutive differences, values for BS-90 snails were compared to baselines from corresponding $\mathrm{M}$ line snail groups. Within each snail strain, values for S. mansoni-exposed snails were compared to baselines from unexposed control snails timematched to the same exposed group (Additional file 4: Table S3).

\section{Phylogenetic and sequence analysis}

Based on InterProScan scanning results, AIG1 domain sequences were extracted from protein sequences of $B$. glabrata, and aligned using the accurate L-INS-i method [72] imbedded in the MAFFT's program [73] through the JABAWS platform [74]. AIG1 domain sequences of Pomacea canaliculata (Pc_XP_025083382.1, Pc_XP_025083383.1, and Pc_XP_025111428.1) were included in the analysis as an outgroup. Additional sequences of AIG1 domains from selected eukaryote species, including Arabidopsis thaliana (thale cress), Branchiostoma floridae (the Florida lancelet), Danio rerio (zebrafish), Homo sapiens (human), Physcomitrella patens (spreading earthmoss), and Saccoglossus kowalevskii (acorn worm), were obtained from Additional files 7 \& 8 in Weiss et al. (2013). Integrity of AIG1 domains was checked and only ones with all G1-G4, CB and G5/IAN motifs were kept for phylogenetic tree construction. Multiple sequence alignment was visualized and sequences were manually trimmed with the multiple sequence alignment view editor Jalview v2.10.5 [75]. Maximum likelihood phylogeny was inferred by running IQ-TREE v 1.6.10 [76], using the best model search with 1000 bootstrap search replicates, and using standard model selection followed by tree inference. The tree was viewed with FigTree v1.4.4 (http://tree. bio.ed.ac.uk/software/figtree/). Similarly, phylogenetic analysis and visualization was applied to both B. glabrata and nine additional molluscan genomes for which AIG1 domains were present, with Acropora millepora as an outgroup.

\section{Other analysis}

Signal peptide search was performed using SignalP 4.0 [77]. Table summary and figure generation were performed with the $\mathrm{R}$ statistical computing environment [78] and Bioconductor [1], including the following packages: ggplot2 [79], reshape2 [80], magic 2.0 (https://docs. ropensci.org/magick/index.html), ggtree 3.9 [81], and openxlsx v4.1.0.1 [82]. Intermediate data analysis was done with an in-house parallel pipeline facilitated with the GNU Parallel tool [71]. 


\section{Supplementary information}

Supplementary information accompanies this paper at https://doi.org/10. 1186/s12864-020-6534-z

Additional file 1: Figure S1. Genome-wide locations of AIG footprints in the B. glabrata BB02. The evolutionary footprints of AIG genes in B. glabrata contain three types: GIMAP (AIG gene with coiled-coil domain), AIG gene without coiled-coil domain, and partial AIG. Scaffold backbones were drawn with gray lines. Scaffolds longer than the figure region were marked with gray dots on left or right end of the gray lines. Genes on the forward strand were marked out using left-to-right arrows above scaffold lines, showing GIMAP genes (blue) and AIG genes (sky blue). Genes on the reverse strand were marked out using right-to-left arrows below scaffold lines, showing GIMAP genes (red) and AIG genes (pink). Partial AIGs (black) were showing on both forward and reverse strand. Scaffold IDs were labeled above each scaffold. Numbers in parenthesis after scaffold IDs are total number of AIG genes (with and without coiled-coils) on the scaffold. Gray parentheses enclosed genes within the same tandem array (no other genes in between).

Additional file 2: Table S1. Consensus sequences of motifs within AIG1 domain among selected organisms. G1, G2, G3, CB, G4, G5/IAN are conserved motifs within AIG1 domain. The consensus sequences of these motifs in different organisms may differ or be absent.

Additional file 3: Table S2. Features of the predicted AIG footprint in B. glabrata The $111 \mathrm{AIG}$ genes (complete and incomplete) and the 148 proteins were detailed, with types of AIG gene, predicted domain architecture, coiled-coil, multimer forms, and genome location.

Additional file 4: Table S3. Overview of the 14 snail groups considered and sequencing information for the RNA-Seq study Two strains of $B$. glabrata snails were used for this study: schistosome-resistant BS-90 and -susceptible $M$ line snails were sampled at 0.5, 2-, 8- and 40-day postexposure to S. mansoni. Time-matched unexposed BS-90 and M line were sampled as control for differential expression analysis. Totals of $3 \sim 6$ biological replicates for each group with raw reads (9-18 million reads/ snail sample) were obtained.

Additional file 5: Table S4. Summary of AIG gene expression in Biomphalaria species from all related studies There are five relevant expression datasets available. I). AIG genes expression in 12 different organs from unstimulated BB02 B. glabrata; II). a microarray study of schistosome resistant BS-90 B. glabrata injected with different PAMPs, including LPS, PGN, and FCN; III). An RNA-Seq transcriptomics study for field-derived Kenyan B. pfeifferi exposed for 1, 3, or 30+ days to $S$. mansoni, relative to unexposed controls; IV). An RNA-Seq study for $S$. mansoni-resistant BS-90 snails relative to susceptible $M$ line snails at constitutive level (no exposure to S. mansoni), and $V$ ) for both $M$ line and BS-90- snails, intrastrain comparisons of snails exposed for $0.5,2,8$ - and 40-day to S. mansoni, relative to time-matched unexposed controls.

Additional file 6: File S1. Multiple sequence alignment of $\mathrm{AIG1}$ domains from selected eukaryotes.

\section{Abbreviations}

AIG1: AvrRpt2-induced gene; ASW: Artificial spring water; CARD: Caspase activation and recruitment domain; CB: Conserved box; DD: Death domain; DE: Differential expression; Dpe: Day post-exposure; FCN: Fucoidan; GIMAP: GTPases of immunity associated proteins; IAN: Immune-associated nucleotide-binding proteins; LPS: Lipopolysaccharide; ML: Maximum likelihood; PAMPs: Pathogen associated molecular patterns; PGN: Peptidoglycan; PPDE: Posterior probability of differential expression; TM: Transmembrane domain; TPM: Transcripts per million

\section{Acknowledgments}

We would like to thank the University of New Mexico Center for Advanced Research Computing, supported in part by the National Science Foundation, for hosting the high-performance computing and large-scale storage resources used in this work. We are grateful to Dr. Sara Brant for her help with clarification of phylogenetic questions. We thank Mrs. Erin Watson for maintenance of the life cycle of regular Biomphalaria glabrata BS-90 and M line. We thank Mr. George Rosenberg for library preparation and sequencing assistance. We are grateful to Dr. James Hane and two anonymous reviewers for helpful comments on the manuscript.

\section{Authors' contributions}

$E S L, L B, L L$ and $S M Z$ conceived and designed the experiments. $L L$ conducted the RNA-Seq experimental work. $L B$ and $L L$ analyzed the data. ESL, LB, LL, SMZ and SKB carried out the interpretation of the data. ESL, LB and LL drafted the manuscript and revised the draft paper. All authors read and approved the final manuscript.

\section{Funding}

This research was funded by NIH awards R37Al101438 and R21Al132953. This research was also facilitated by to the Center for Evolutionary and Theoretical Immunology (CETI) supported by NIH COBRE award 5P30GM110907-05. Funding agency played no role in the study design, data analysis and interpretation, or in writing the manuscript.

\section{Availability of data and materials}

The raw RNA sequencing data are available at NCBI under SRA accession: PRJNA591872. All other data generated or analyzed during this study are included in this published article and its supplementary information files.

Ethics approval and consent to participate

Not applicable.

\section{Consent for publication}

Not applicable.

\section{Competing interests}

The authors declare that they have no competing interests.

\section{Author details}

${ }^{1}$ Center for Evolutionary and Theoretical Immunology, Department of Biology, University of New Mexico, Albuquerque, NM 87131, USA. ${ }^{2}$ Wellcome Sanger Institute, Wellcome Trust Genome Campus, Hinxton CB10 1SA, UK.

Received: 14 September 2019 Accepted: 23 January 2020 Published online: 02 March 2020

\section{References}

1. Gentleman RC, Carey VJ, Bates DM, Bolstad B, Dettling M, Dudoit S, et al. Bioconductor: open software development for computational biology and bioinformatics. Genome Biol. 2004:5:R80.

2. Aguilar R, Jedlicka AE, Mintz M, Mahairaki V, Scott AL, Dimopoulos G. Global gene expression analysis of Anopheles gambiae responses to microbial challenge. Insect Biochem Mol Biol. 2005;35:709-19.

3. Deleury E, Dubreuil G, Elangovan N, Wajnberg E, Reichhart JM, Gourbal B, et al. Specific versus non-specific immune responses in an invertebrate species evidenced by a comparative de novo sequencing study. PLoS One. 2012;7(3):e32512.

4. Hibino T, Loza-Coll M, Messier C, Majeske AJ, Cohen AH, Terwilliger DP, et al The immune gene repertoire encoded in the purple sea urchin genome. Dev Biol. 2006;300:349-65.

5. Zhang L, Li L, Guo X, Litman GW, Dishaw LJ, Zhang G. Massive expansion and functional divergence of innate immune genes in a protostome. Sci Rep. 2015;5:1-11.

6. Leulier F, Lemaitre B. Toll-like receptors - taking an evolutionary approach. Nat Rev Genet. 2008;9:165-78.

7. Messier-Solek C, Buckley KM, Rast JP. Highly diversified innate receptor systems and new forms of animal immunity. Semin Immunol. 2010;22: 39-47.

8. Ghosh J, Lun CM, Majeske AJ, Sacchi S, Schrankel CS, Smith LC. Invertebrate immune diversity. Dev Comp Immunol. 2011;35:959-74. https://doi.org/10. 1016/j.dci.2010.12.009

9. Liberti A, Leigh B, De Santis R, Pinto MR, Cannon JP, Dishaw $\sqcup$, et al. An immune effector system in the protochordate gut sheds light on fundamental aspects of vertebrate immunity. Results Probl Cell Differ. 2015; 51:159-73.

10. Zhang S-M, Adema CM, Kepler TB, Loker ES. Diversification of Ig superfamily genes in an invertebrate. Science. 2004;305:251-4. 
11. Loker ES. Macroevolutionary immunology: a role for immunity in the diversification of animal life. Front Immunol. 2012;3:25. https://doi.org/10. 3389/fimmu.2012.00025.

12. Hoffmann JA, Kafatos FC, Janeway CA, Ezekowitz RAB. Phylogenetic perspectives in innate immunity. Science. 1999;284(80):1313-8.

13. Sijmons PC, Grundler FMW, von Mende N, Burrows PR, Wyss U. Arabidopsis thaliana as a new model host for plant-parasitic nematodes. Plant J. 1991;1: 245-54.

14. Katagiri F, Thilmony R, He SY. The Arabidopsis thaliana-pseudomonas syringae interaction. Arab B. 2002;1:e0039.

15. Kai M, Vespermann A, Piechulla B. The growth of fungi and Arabidopsis thaliana is influenced by bacterial volatiles. Plant Signal Behav. 2008;3:482-4.

16. Nitta T, Takahama Y. The lymphocyte guard-IANs: regulation of lymphocyte survival by IAN/GIMAP family proteins. Trends Immunol. 2007;28:58-65.

17. Dalberg U, Markholst H, Hornum L. Both Gimap5 and the diabetogenic BBDP allele of Gimap5 induce apoptosis in T cells. Int Immunol. 2007;19:447-53.

18. Yano K, Carter C, Yoshida N, Abe T, Yamada A, Nitta T, et al. Gimap3 and Gimap5 cooperate to maintain T-cell numbers in the mouse. Eur J Immunol. 2014;44:561-72.

19. Weiss Y, Foret S, Hayward DC, Ainsworth T, King R, Ball EE, et al. The acute transcriptional response of the coral Acropora millepora to immune challenge: expression of GiMAP/IAN genes links the innate immune responses of corals with those of mammals and plants. BMC Genomics. 2013;14:400.

20. Kim CY, Zhang X, Witola H. Small GTPase immunity-associated proteins mediate resistance to Toxoplasma gondii infection in Lewis rat. Infect Immun. 2018;86:e00582-17.

21. Reuber TL, Ausubel FM. Isolation of Arabidopsis genes that differentiate between resistance responses mediated by the RPS2 and RPM1 disease resistance genes. Plant Cell. 1996:8:241-9.

22. Biller L, Davis PH, Tillack M, Matthiesen J, Lotter H, Stanley SL, et al. Differences in the transcriptome signatures of two genetically related Entamoeba histolytica cell lines derived from the same isolate with different pathogenic properties. BMC Genomics. 2010;11(1):63.

23. McDowell IC, Modak TH, Lane CE, Gomez-chiarri M. Multi-species protein similarity clustering reveals novel expanded immune gene families in the eastern oyster Crassostrea virginica. Fish Shellfish Immunol. 2016;53:13-23. https://doi.org/10.1016/j.fsi.2016.03.157.

24. Zhang S-M, Loker ES, Sullivan JT. Pathogen-associated molecular patterns activate expression of genes involved in cell proliferation, immunity and detoxification in the amebocyte-producing organ of the snail Biomphalaria glabrata. Dev Comp Immunol. 2016;56:25-36.

25. Buddenborg SK, Bu L, Zhang S-M, Schilkey FD, Mkoji GM, Loker ES. Transcriptomic responses of Biomphalaria pfeifferi to Schistosoma mansoni: Investigation of a neglected African snail that supports more S. mansoni transmission than any other snail species. PLoS Negl Trop Dis. 2017;11: e0005984. https://doi.org/10.1371/journal.pntd.0005984.

26. Zhang G, Fang X, Guo X, Li L, Luo R, Xu F, et al. The oyster genome reveals stress adaptation and complexity of shell formation. Nature. 2012;490:49-54.

27. Guerin MN, Weinstein DJ, Bracht JR. Stress adapted Mollusca and Nematoda exhibit convergently expanded Hsp70 and AIG1 gene families. J Mol Evol. 2019:87(9-10):289-97. https://doi.org/10.1007/s00239-019-09900-9.

28. Liu C, Wang T, Zhang W, Li X. Computational identification and analysis of immune-associated nucleotide gene family in Arabidopsis thaliana. J Plant Physiol. 2008;165:777-87.

29. Krücken J, Schroetel RMU, Müller IU, Saïdani N, Marinovski P, Benten WPM, et al. Comparative analysis of the human gimap gene cluster encoding a novel GTPase family. Gene. 2004;341:291-304.

30. Nitta T, Nasreen M, Seike T, Goji A, Ohigashi I, Miyazaki T, et al. IAN family critically regulates survival and development of T lymphocytes. PLoS Biol. 2006:4:593-605

31. Wang Z, Li X. IAN/GIMAPs are conserved and novel regulators in vertebrates and angiosperm plants. Plant Signal Behav. 2009;4:165-7.

32. Colley DG, Bustinduy AL, Secor WE, King CH. Human schistosomiasis. Lancet. 2014;383:2253-64. https://doi.org/10.1016/S0140-6736(13)61949-2.

33. Hotez PJ, Alvarado M, Basáñez MG, Bolliger I, Bourne R, Boussinesq M, et al. The global burden of disease study 2010: interpretation and implications for the neglected tropical diseases. PLoS Negl Trop Dis. 2014;8(7):e2865.

34. Truebestein L, Leonard TA. Coiled-coils: the long and short of it. BioEssays. 2016:38:903-16.
35. Gorbushin AM. Derivatives of the lectin complement pathway in Lophotrochozoa. Dev Comp Immunol. 2019;94:35-58. https://doi.org/10. 1016/j.dci.2019.01.010

36. Adema CM, Hillier LW, Jones CS, Loker ES, Knight M, Minx P, et al. Whole genome analysis of a schistosomiasis-transmitting freshwater snail. Nat Commun. 2017:8:1-11.

37. Kocot KM, Cannon JT, Todt C, Citarella MR, Kohn AB, Meyer A, et al. Phylogenomics reveals deep molluscan relationships. Nature. 2011;477:4526. https://doi.org/10.1038/nature10382.

38. Smith SA, Wilson NG, Goetz FE, Feehery C, Andrade SCS, Rouse GW, et al. Resolving the evolutionary relationships of molluscs with phylogenomic tools. Nature. 2011;480:364-7.

39. Liu C, Zhang Y, Ren Y, Wang H, Li S, Jiang F, et al. The genome of the golden apple snail Pomacea canaliculata provides insight into stress tolerance and invasive adaptation. Gigascience. 2018;7:1-13.

40. Weber $\mathrm{CH}$, Vincenz $\mathrm{C}$. The death domain superfamily: a tale of two interfaces? Trends Biochem Sci. 2001;26:475-81.

41. Park HH, Lo Y-C, Lin S-C, Wang L, Yang JK, Wu H. The death domain superfamily in intracellular signaling of apoptosis and inflammation. Annu Rev Immunol. 2007;25:561-86.

42. Hofmann K, Bucher P, Tschopp J. The CARD domain: a new apoptotic signalling motif. Trends Biochem Sci. 1997;22:155-6.

43. Jiang C, Lin X. Regulation of NF-kB by the CARD proteins. Immunol Rev. 2012;246:141-53.

44. Groves MR, Barford D. Topological characteristics of helical repeat proteins. Curr Opin Struct Biol. 1999;9:383-9.

45. Gul I, Hulpiau P, Saeys Y, Van RF. Metazoan evolution of the armadillo repeat superfamily. Cell Mol Life Sci. 2017;74:525-41.

46. Perler FB. Protein splicing of inteins and hedgehog autoproteolysis: structure, function, and evolution. Cell. 1998;92:1-4.

47. Pan D, Zhang L. Tandemly arrayed genes in vertebrate genomes. Comp Funct Genomics. 2008:2008:545269.

48. Graham GJ. Tandem genes and clustered genes. J Theor Biol. 1995;175:71-87.

49. DeJong RJ, J.a M, Paraense WL, Pointier JP, Amarista M, Ayeh-Kumi PF, et al. Evolutionary relationships and biogeography of Biomphalaria (Gastropoda: Planorbidae) with implications regarding its role as host of the human bloodfluke, Schistosoma mansoni. Mol Biol Evol. 2001;18:2225-39.

50. Abe M, Kuroda R. The development of CRISPR for a mollusc establishes the formin Lsdia1 as the long-sought gene for snail dextral/sinistral coiling. Development. 2019;146:dev175976. doi:https://doi.org/10.1242/dev.175976.

51. Giraldo-Calderón Gl, Emrich SJ, MacCallum RM, Maslen G, Dialynas E, Topalis $P$, et al. VectorBase: an updated bioinformatics resource for invertebrate vectors and other organisms related with human diseases. Nucleic Acids Res. 2015;43:D707-13.

52. O'Leary NA, Wright MW, Brister JR, Ciufo S, Haddad D, McVeigh R, et al. Reference sequence (RefSeq) database at NCBI: current status, taxonomic expansion, and functional annotation. Nucleic Acids Res. 2016;44:D733-45.

53. Resource CN, Sayers EW, Agarwala R, Bolton EE, Brister JR, Canese K, et al. Database resources of the National Center for Biotechnology Information. Nucleic Acids Res. 2019:47:D23-8.

54. Finn RD, Coggill P, Eberhardt RY, Eddy SR, Mistry J, Mitchell AL, et al. The Pfam protein families database: towards a more sustainable future. Nucleic Acids Res. 2016;44:D279-85.

55. Jones P, Binns D, Chang H, Fraser M, Li W, Mcanulla C, et al. InterProScan 5 : genome-scale protein function classification. Bioinformatics. 2014;30:1236-40.

56. Marchler-Bauer A, Lu S, Anderson JB, Chitsaz F, Derbyshire MK, DeWeeseScott C, et al. CDD: a conserved domain database for the functional annotation of proteins. Nucleic Acids Res. 2011;39(SUPPL. 1):225-9.

57. Marchler-Bauer A, Bryant SH. CD-Search: Protein domain annotations on the fly. Nucleic Acids Res. 2004, 32(WEB SERVER ISS):327-31.

58. Krogh A, Larsson Ė, Von Heijne G, Sonnhammer ELL. Predicting transmembrane protein topology with a hidden Markov model : application to complete genomes. J Mol Biol. 2001;305:567-80.

59. Lupas A, Van DM, Stock J. Predicting coiled coils from protein sequences. Science. 1991;252(80):1162-4.

60. Vincent TL, Green PJ, Woolfson DN. LOGICOIL - multi-state prediction of coiled-coil oligomeric state. Bioinformatics. 2013;29:69-76.

61. Bolger AM, Lohse M, Usadel B. Trimmomatic: a flexible trimmer for Illumina sequence data. Bioinformatics. 2014;30:2114-20. 
62. Dobin A, Davis CA, Schlesinger F, Drenkow J, Zaleski C, Jha S, et al. STAR: ultrafast universal RNA-seq aligner. Bioinformatics. 2013;29:15-21.

63. Pertea M, Pertea GM, Antonescu CM, Chang TC, Mendell JT, Salzberg SL. StringTie enables improved reconstruction of a transcriptome from RNA-seq reads. Nat Biotechnol. 2015;33:290-5.

64. Kolde R. Pheatmap: pretty heatmaps. R package version. 2012;61:617.

65. Loker ES, Hertel LA. Alterations in Biomphalaria glabrata plasma induced by infection with the digenetic trematode Echinostoma paraensei. J Parasitol. 1987;73:503-13.

66. Stibbs HH, Owczarzak A, Bayne CJ, DeWan P. Schistosome sporocyst-killing amoebae isolated from Biomphalaria glabrata. J Invertebr Pathol. 1979;33: $159-70$

67. Lu L, Zhang S-M, Mutuku MW, Mkoji GM, Loker ES. Relative compatibility of Schistosoma mansoni with Biomphalaria sudanica and B. pfeifferi from Kenya as assessed by PCR amplification of the S. mansoni ND5 gene in conjunction with traditional methods. Parasit Vectors. 2016;9:166.

68. Liao Y, Smyth GK, Shi W. FeatureCounts: an efficient general purpose program for assigning sequence reads to genomic features. Bioinformatics. 2014:30(7):923-30.

69. Leng N, Dawson JA, Thomson JA, Ruotti V, Rissman Al, Smits BMGG, et al. EBSeq : an empirical Bayes hierarchical model for inference in RNA-seq experiments. Bioinformatics. 2013;29:1035-43.

70. Varet H, Brillet-Guéguen L, Coppée JY, Dillies MA. SARTools: a DESeq2- and EdgeR-based R pipeline for comprehensive differential analysis of RNA-Seq data. PLoS One. 2016;11:e0157022.

71. Tange O. GNU parallel: the command-line power tool. USENIX Mag. 2011;36: 42-7. https://doi.org/10.5281/zenodo.16303.

72. Berger MP, Munson PJ. A novel randomized iterative strategy for aligning multiple protein sequences. Bioinformatics. 1991;7:479-84.

73. Katoh $\mathrm{K}$, Toh H. Parallelization of the MAFFT multiple sequence alignment program. Bioinformatics. 2010;26:1899-900.

74. Troshin PV, Procter JB, Sherstnev A, Barton DL, Madeira F, Barton GJ. JABAWS 2.2 distributed web services for Bioinformatics: Protein disorder, conservation and RNA secondary structure. Bioinformatics. 2018;34:1939-40.

75. Waterhouse AM, Procter JB, Martin DMA, Clamp M, Barton GJ. Jalview version 2-a multiple sequence alignment editor and analysis workbench. Bioinformatics. 2009;25:1189-91.

76. Nguyen LT, Schmidt HA, Von Haeseler A, Minh BQ. IQ-TREE: a fast and effective stochastic algorithm for estimating maximum-likelihood phylogenies. Mol Biol Evol. 2015;32:268-74.

77. Brunak S, Petersen TN, Brunak S, von Heijne G, Nielsen H. SignalP 4.0: discriminating signal peptides from transmembrane regions. Nat Methods. 2011;8:785-6,

78. R Core Team. R: a language and environment for statistical computing Vienna R Foudation Stat Comput. 2013.

79. Wickham H. ggplot: elegant graphics for data analysis. Springer; 2016 .

80. Wickham H. Reshape2: a reboot of the reshape package. R package version 2015. https://github.com/hadley/reshape.

81. Yu G, Smith DK, Zhu H, Guan Y, Lam TTY. GGTREE: an R package for visualization and annotation of phylogenetic trees with their covariates and other associated data. Methods Ecol Evol. 2017;8:28-36.

82. Walker A, Braglia L. openxIsX: read, write and edit XLSX files. R package version 3.0.0. 2015.

\section{Publisher's Note}

Springer Nature remains neutral with regard to jurisdictional claims in published maps and institutional affiliations.

Ready to submit your research? Choose BMC and benefit from:

- fast, convenient online submission

- thorough peer review by experienced researchers in your field

- rapid publication on acceptance

- support for research data, including large and complex data types

- gold Open Access which fosters wider collaboration and increased citations

- maximum visibility for your research: over $100 \mathrm{M}$ website views per year

At BMC, research is always in progress.

Learn more biomedcentral.com/submissions 